\title{
Study of Ergonomic Workstations and its Relationship with the Prevalence of Upper Limbs Disorders and Psychosocial Factors in the Staff of Lorestan Province Banks
}

Shahnaz Tabatabaei, Associate professor of Ergonomics Department, School of Public Health and Safety Shahid Beheshti University of Medical Sciences, Tehran, Iran.

[1 Mostafa Hasanvand, ( ${ }^{\star}$ Corresponding author), MSc Student of Ergonomics, School of Public Health and Safety Shahid Beheshti University of Medical Sciences, Tehran, Iran. mostafa.hasanvand85@gmail.com

\section{Abstract}

Background and aims: The bank staff are often exposed to repeated movements, awkward posture and working by hand. Also, they encounter many psychosocial problems at their workplace. Therefore, this research was conducted with the aim of studying the ergonomic workstations and its relationship with the prevalence of upper limbs disorders and its psychosocial factors on the staff of Lorestan province banks.

Methods: This was a practical and descriptive- correlative research. The statistical population consisted of 900 bank employees of Lorestan province, with a sample population of 275 , who were selected using the stratified cluster sampling method. Data was collected by these questionnaires: Demographic; Ergonomic Checklist of Occupational Safety and Health Administration (OSHA); Nordic (Kuorinka, 1987); and Psychosocial Factors (Hersey and Goldsmith 1980). For the analysis of data (t-test and multiple regression) SPSS 23, was used.

Results: The highest prevalence of upper limbs disorder was observed in the neck $(61.5 \%)$ and the shoulder (53.8\%). The mean of psychosocial factors in the bank staff was found to be a little more than average. It was found to be a significant difference between means of the sample group in psychosocial factors with respect of their evaluation of physical and mental work condition ( $\alpha$ $\leq 0.01$ ). There was a significant and negative correlation between all ergonomic indexes with upper limbs disorders and psychosocial factors followed by these disorders $(\mathrm{p} \leq 0.01)$.

Conclusion: The psychosocial condition of the staff is on the average. However, managers should be aware of the needs of the staff. Furthermore, to work efficiently, the staff need to perceive social support, motivation, and feedback from their supervisors.

Conflicts of interest: The authors declare that there are no conflicts of interest regarding the publication of this manuscript.

Funding: None

\section{Keywords}

Ergonomic workstation

Upper limbs disorders

Psychosocial factors

Bank staff

Received: 2018/06/24

Accepted : 2019/09/13 


\section{INTRODUCTION}

Organizations have considered human resources as the most important source to gain competitive advantages. Besides, there is a competition to provide welfare plans and pay attention to staff. On the one hand, using ergonomics is followed up by increasing safety, efficiency, individuals' comfort, and organizing the work environment, according to the abilities of official users. On the other hand, musculoskeletal disorders are considered as the main problem regarding career health, and it can be effective on the quality of work life, medical expenses, efficiency, production and productivity at workplace. Staff in general, and especially the bank staff are often exposed to repeated movements, awkward posture and working by hand, which are risk factors for musculoskeletal symptoms. Furthermore, staff encounter many psychosocial problems such as stress as a result of work time and stressful work conditions. Although, the role of psychosocial factors has been acknowledged on the development of musculoskeletal symptoms. Musculoskeletal symptoms have been reported among bank staff. These symptoms can lead to MSDs, if the symptom treatment and recovery is not considered. Many factors are related to the emerge of MSDs, like demographical variables (age, gender, weight and height), individual lifestyle choices (smoking and doing exercise), physical aspects of work (duration of working on computer, computer work skills, awkward posture, repeated movements, and having or not having regular rest times), and psychological aspects of work (emotional exhaustion and the job security). In addition, some studies have shown that there is relationship between musculoskeletal disorders and obesity. Sociodemographic variables such as age, gender, race, income level, employment status, and geographic situation have a significant relationship with the MSDs. In respect of gender, MSDs was reported more among women compered to men, and it can be related to the tolerance of pain in men and the fact that they rarely refer to clinics. Some studies have shown the positive effects of doing exercise on reducing MSDs in upper limbs such as neck and shoulder. Kaliniene and others (2013), have found that the Body Mass Index (BMI), has a significant relationship with MSDs. Tantawy et al. (2017), reported that individuals who are overweight have reported more MS pains, compared to people who are fit or underweight, while Soleiman and others (2017), reported that there is no significant relationship between BMI and MSDs. In a study by Yeow and Sen (2003), they concluded that the nature and type of work in a bank, can be the reason for the occurrence of MSDs. Saklani and Jha (2014), reported that there are some risk factors that affect the emergence of MSDs, like static body posture for a long time, awkward posture that is caused by unsuitable design of workstations, and the use of nonergonomic desks and chairs. Helland and others (2011), believed that one of the most important problem that ergonomists have to deal with, is MSDs, which is the most common disorder among the bank staff. In a research by Mahmud et al. (2014), the main reason of office workers' MSDs, could be related to sitting on the chair for a long time to work on the computer, awkward posture, doing repeated work such as typing and the incorrect use of the mouse. Bernard and others (1997), reported the relationship between the ergonomic factors and evaluating workstations and MSDs. In a research conducted by Falaki and others (2016), results indicated the prevalence of MSDs among staff because of their awkward posture, and they suggested the ergonomic improvement of the workplace. Another study by Tabatabaei Yahyaabadi and others (2017), reported the incidence of MSDs in back, neck and shoulder among the bank staff, and a negative relationship between MSDs and overall ergonomic index. In addition, Tabatabaei et al. (2017) and Ghasemzade and others (2018), reported that there is relationship between the workstations ergonomic indexes and prevalence of MSDs, with the quality of life and quality of work life in office staff. Another research by Choobineh and others (2011), revealed that there is a correlation between the high levels of Body Mass Index (BMI) and MSDs; and working overtime and upper limbs disorders, in the administrative workplace. Therefore, considering the information and evidences which are mentioned in this research, and the effects of ergonomics on human's working life, this research was conducted with the aim of studying the ergonomic workstations and its relation with prevalence of upper limbs disorders and psychosocial factors in the staff of Lorestan province banks. In this article, in addition to the study of ergonomic workstations of banks and prevalence of upper limbs disorders, the relationship between the both variables and the psychosocial factors of the work environment is considered. Since the relationship between the 
three variables has been rarely studied by other researchers, conducting the present research was so important and necessary.

\section{METHODS AND MATERIALS}

This was a practical and descriptive-correlative research. The statistical population consisted of 900 employees of banks in Lorestan province, with a sample of 275 , whom were selected using stratified cluster sampling. For data collection the following tools were used: Demographic questionnaire including items regarding age, gender, work experience, work hours, physical and mental aspects of work; Ergonomic Checklist of Occupational Safety and Health Administration (OSHA): This research used the ergonomic checklist which was already designed to assess the ergonomic factors of the office workstations. In fact, this checklist was provided based on reviewing the available checklists, the office workplace ergonomic evaluation checklist which was published by OSHA, consultation with experts, and interviewing the bank employees. Therefore, it is a comprehensive checklist that consists of 78 items and six subscales: 1 . Working Posture, 2. Equipment, 3. Layout and Design, 4. Environmental Condition, 5. Overall Atmosphere of the Bank and 6. Ergonomics Application. The checklist items have three options to answer: "Yes" (1), "no" (0), "not applicable" (0), the high score shows desirable ergonomic workstation and low score reveals undesirable ergonomic workstation in banks. To evaluate the ergonomic workstations of banks by evaluator: first, all the workstations were determined, afterwards, the lists of workstations were provided and lastly, the evaluations of workstations were conducted; General Form of Musculoskeletal Disorder Nordic questionnaire (Kuorinka,1987); and Psychosocial Factors questionnaire (Hersey and Goldsmith, 1980), which consists of 26 items and 7 subscales: 1. Ability, 2. Perception and cognition, 3. Organizational support, 4. Motivation, 6. Esteem, 7. Adjustment. This five point scale has scored from "very low" (1) to "very high" (5), where the low score shows the high effect of psychosocial factors on the staff and vice versa. In this research, Psychosocial Factors questionnaire has an acceptable constructional reliability. Its Alpha Cronbach is in the range of 0.71 to 0.84 for subscales, and 0.89 for overall scale; for the analysis of data (t-test and multiple regression) the SPSS $_{23}$ was used.

\section{RESULTS}

Descriptive findings showed that the staff were $76.72 \%$ male, $23.28 \%$ female, the age average was 36.36 , the number of costumers 59 per day, the mean of work experience 11.36, work hours 47.89 hours per week, overtime work 9.83 hours weekly, higher education $89.45 \%$ and $78.18 \%$ married. Mean and standard deviation scores for ergonomic workstations indexes were: work posture $=63.64$ (32.50), equipment $=$ 49.33 (34.10), layout and design $=65.68(32.30)$, environmental condition $=38.02(25.78)$, overall atmosphere of banks $=80.40$ (13.98), ergonomic application $=8.72$ (13.38), and overall ergonomic workstations index $=56.70$ (33.96). Therefore, the most desirable ergonomic index was related to overall atmosphere of banks (80.40) and the least desirable of ergonomic index was related to ergonomic application (8.72). The highest incidence of upper limbs disorders was in the neck (61.5\%) and the shoulder (53.8\%). The mean of psychosocial factors in the staff of Lorestan province banks, was found to be a little more than average. It was found to be a significant difference between means of the sample group in psychosocial factors with respect of their evaluation of physical and mental work condition $(\alpha \leq 0.01)$. There was a significant and negative correlation between all ergonomic indexes with the exception of environmental condition with upper limbs disorders, considering the value of $\mathrm{R}_{\text {adj }}^{2}=481$, all the ergonomic indexes with the exception of environmental condition, can predict $48 \%$ of upper limbs disorders variance significantly $(\mathrm{p} \leq 0.01)$, it means, more increase in desirability of ergonomic indexes, less in upper limbs disorders; and it was found to be a significant and negative association between all dimensions of psychosocial factors with the exception of ability dimension with upper limbs disorders, considering the value of $\mathrm{R}_{\text {adj }}^{2}=0.336$, all dimensions of psychosocial factors with the exception of ability dimension can predict $32 \%$ of upper limbs disorders variance significantly $(\mathrm{p} \leq 0.001)$, if there is an increase in psychosocial factors, upper limbs disorders will be decreased and vice versa.

\section{DISCUSSION}

The results of the research shows that the MSDs was observed in the neck, shoulder, wrist, and hand of the staff, which was similar to the results of researches by Kaewdok and 
Taptagaporn (2017), regarding the effects of MSDs on the quality of work life; Janwantanakul and others (2008), related to the incidence of MSDs in office staff; Abledu and Abledu (2012), in the staff of bank; Maiya et al. (2011), in the computer users; Bernaards and others (2011), regarding the effects of exercise on MSDs in computer users; Kaliniene et al. (2013); Mahmud and others (2014); Falaki et al. (2016); and Tabatabaei Yahyaabadi and others (2017). Accordingly, the high prevalence of upper limbs disorders is generally observed in the staff of office and banks, and particularly in computer users. The results of this research reveals that the most undesirable ergonomic indicators were ergonomic application, environmental condition, and tools \& equipment, which was similar to the results of researches by Woods and Buckle (2002); Yeow and Sen (2003); Saklani and Jha (2014); Bernard et al. (1997); and Choobineh and others (2013). In addition, the results of the present research indicate the significant difference between mean of psychosocial factors based on the physical and mental condition of workplace for staff, which was supported by the researches of Mahmud et al. (2014), on the relationship between psychosocial factors, ergonomic risk factors and MSDs in office staff; Ghasemzade and others (2018); and Choobineh et al. (2011).

\section{CONCLUSION}

It can be generally concluded that psychosocial factors of a workplace are due to perception and cognition of staff from their organizational condition. Organizational condition can be included as social support, motivation, feedback to staff regarding their job performances, creating interactive relationship between staff and supervisors, and increasing the awareness of staff about the nature of office and banking works; these factors can play an effective role in creating the adjustment between staff and their workplace. Therefore, organizational condition in various workplaces can provide different psychosocial factors. In addition, the results show that some physical and psychosocial factors, such as awkward work posture, shortage of ergonomic equipment, unsuitable layout and designing, and the lack of using ergonomic principles at workplace, could be the result of unawareness of the staff about ergonomic principles and the way they could be used, lack of budget to provide ergonomic equipment and designing, lack of perception and cognition about the nature of office work by staff, lack of social support at workplace, and inappropriate atmosphere of banks in Lorestan Province. Therefore, all factors could be effective on the prevalence of upper limbs disorders among the staff. Generally, and according to the results of the present research, some improvements can be done by managers for establishing suitable work condition, providing ergonomic equipment and tools, and preventing the upper limbs disorders in the staff of Lorestan province banks. Simultaneously, managers can increase the awareness of staff regarding the nature of office and banking work, ergonomic principles and using them in the banks in order to decrease the upper limbs disorders. As a result, all the mentioned activities can contribute to an increase in the organizational productivity. The limitations of the present research was the lack of control on the variables that were out of the workplace and the lack of generalization of the results to the other banks in other provinces of Iran, since this research was limited to the Lorestan province. Researchers recommend the ergonomic improvements in the banks, educating the ergonomic principles to the staff, and mediating the organizational climate by social support, motivation and feedback.

\section{ACKNOLEDGEMENT}

The authors appreciate all the staff and managers of Lorestan province banks for their cooperation in the present study.

How to cite this article:

Shahnaz Tabatabaei, Mostafa Hasanvand. Study of Ergonomic Workstations and its Relationship with the Prevalence of Upper Limbs Disorders and Psychosocial Factors in the Staff of Lorestan Province Banks. Iran Occupational Health. 2021 (01 Nov);18:21. 

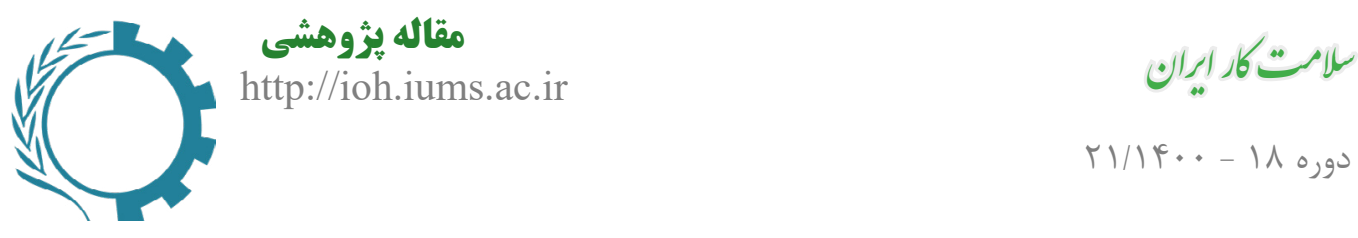

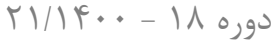

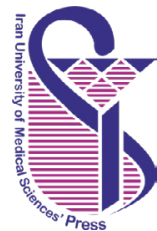

\section{بررسى ارتونوميك ايستكاه هاى كار و ارتباط آن با شيوع اختلالات اندام هاى فوقانى و عوامل روانى - اجتماعى در كاركنان بانك هاى استان لر رستان}

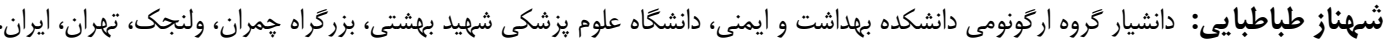

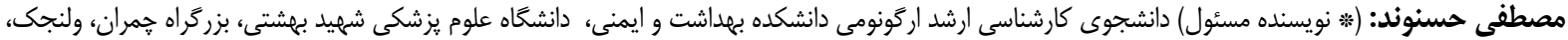

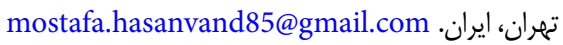
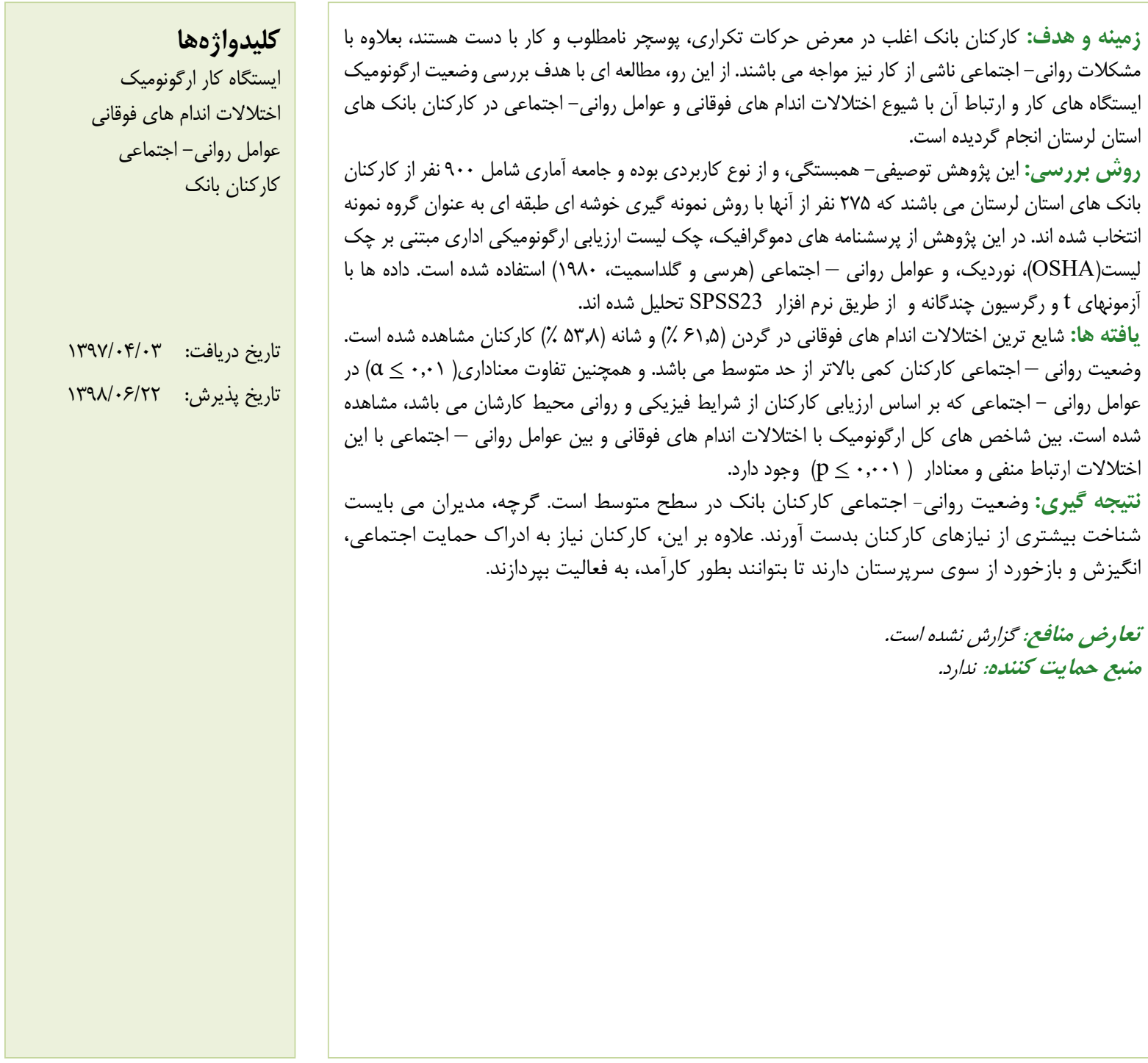

شيوه استناد به اين مقاله:

Shahnaz Tabatabaei, Mostafa Hasanvand. Study of Ergonomic Workstations and its Relationship with the Prevalence of Upper Limbs Disorders and Psychosocial Factors in the Staff of Lorestan Province Banks. Iran Occupational Health. 2021 (01 Nov);18:21. 
مانند گردن و شانه را نشان مى دهند. همجنين شاخص

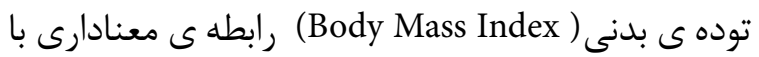

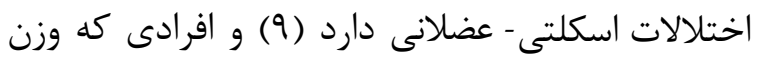

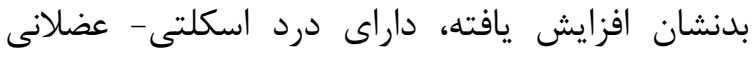

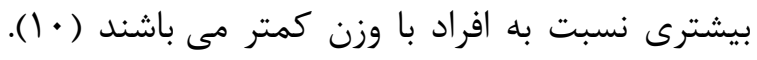

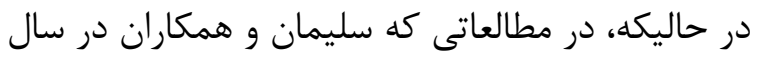

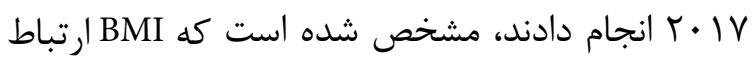

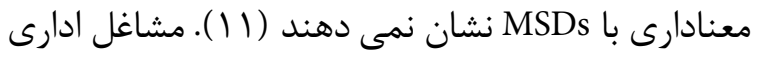

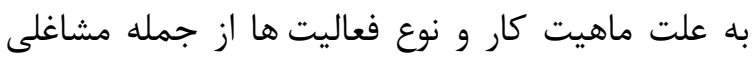

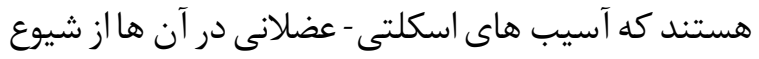

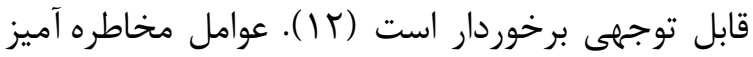

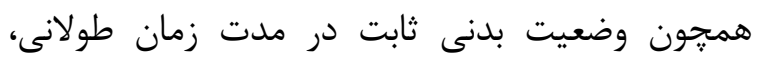

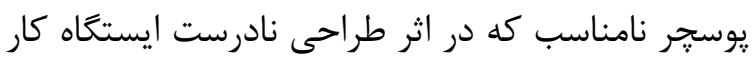

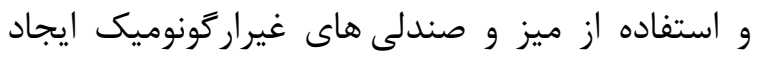

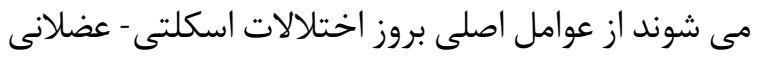

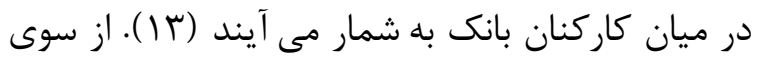

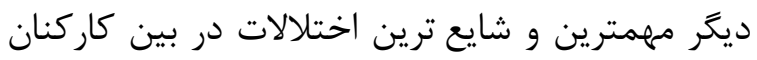

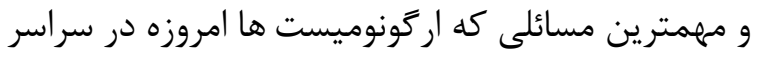

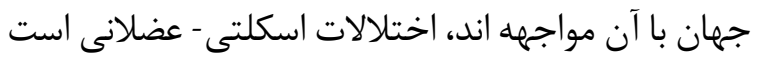

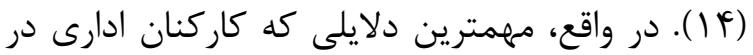

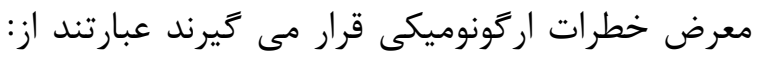

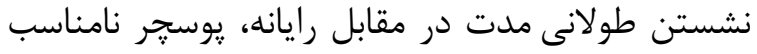

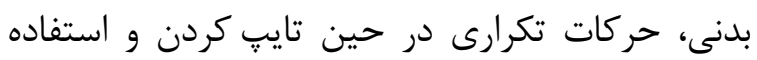

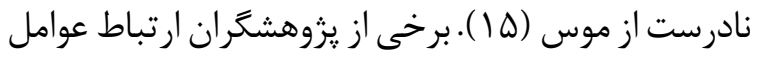

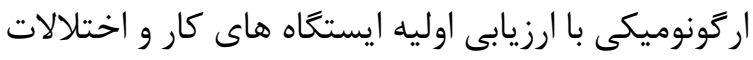

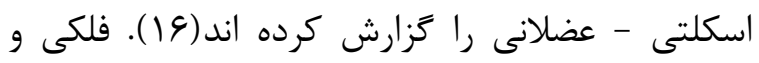

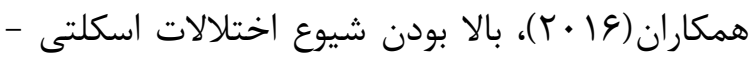

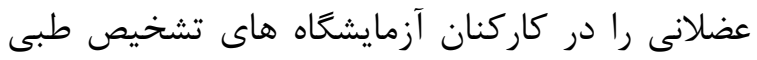

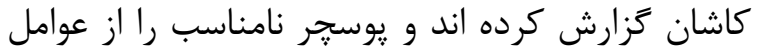

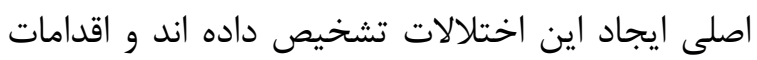

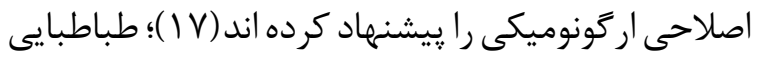

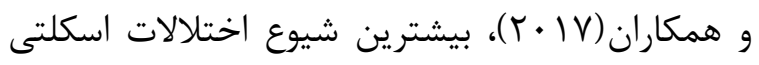

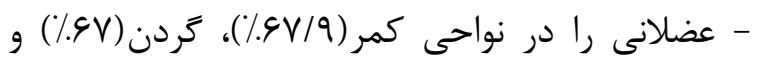

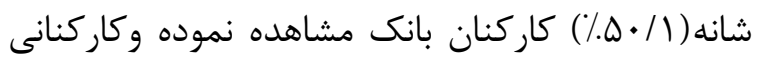

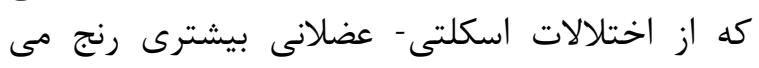

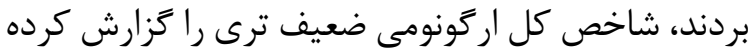

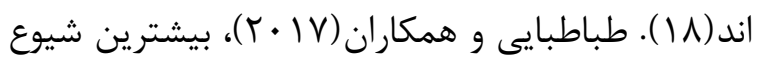

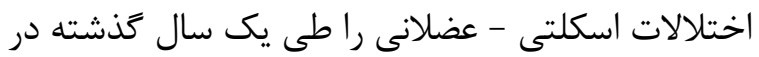

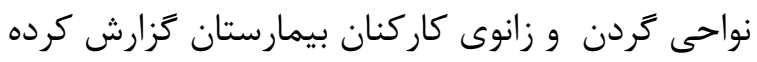

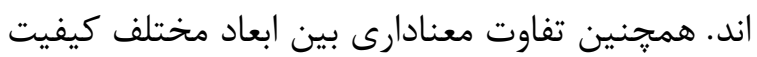

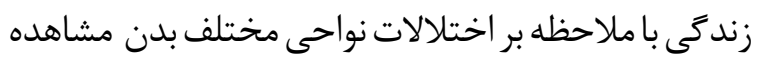

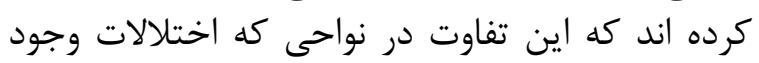

مقدمه

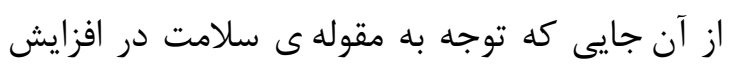

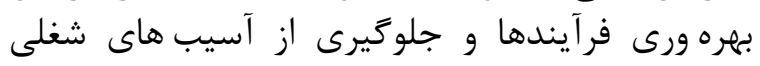

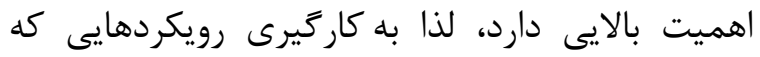

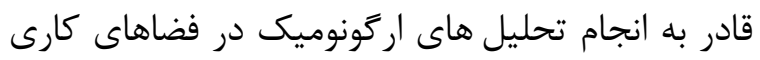

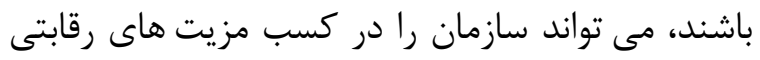

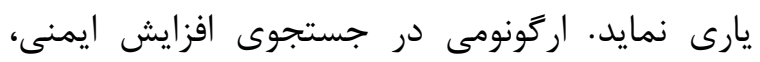

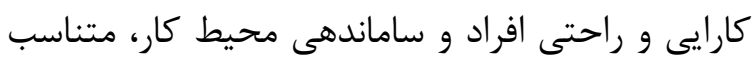

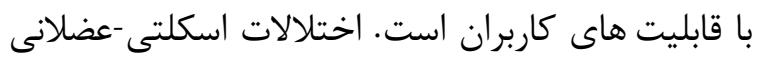

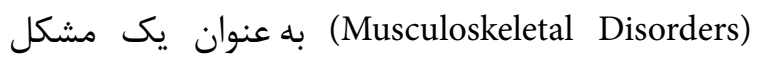
عمده سلامت شغلى محسوب شده و مى تواند بر كيفيت

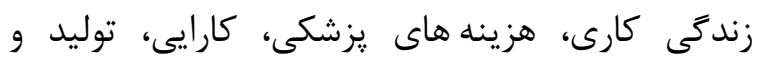

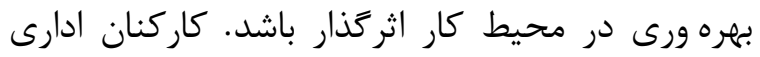

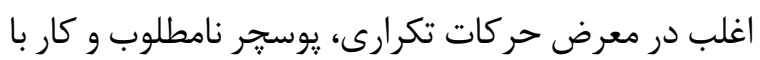

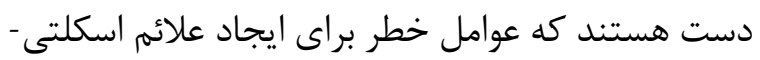

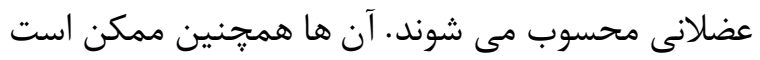

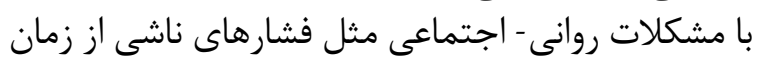

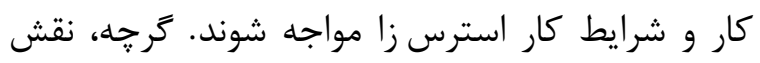

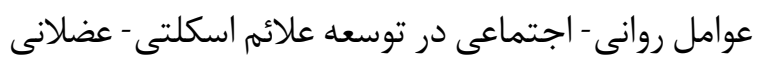
به خوبى شناخته شده است. علائم اسكلتى - عضلانى داعى در

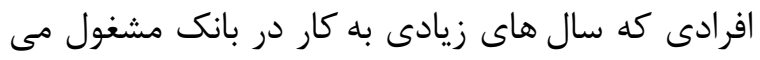

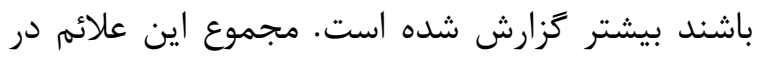

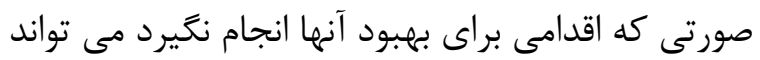

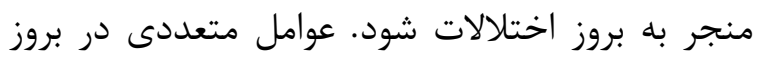

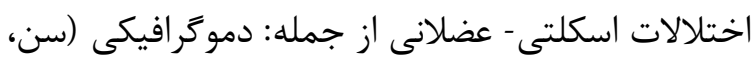

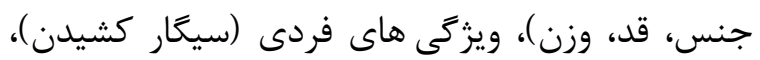

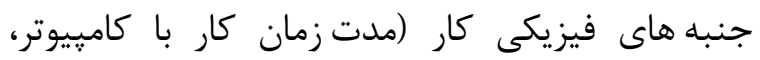

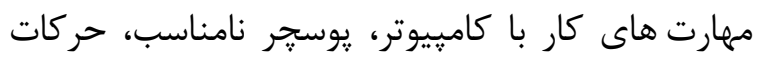

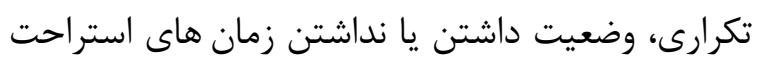

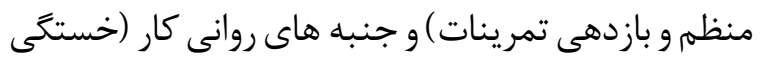

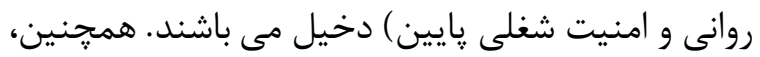

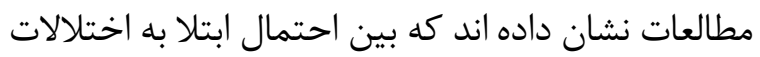

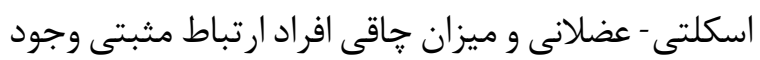

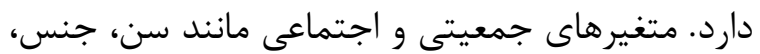

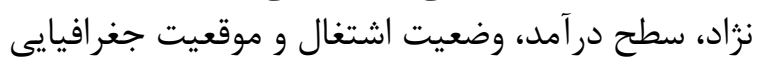

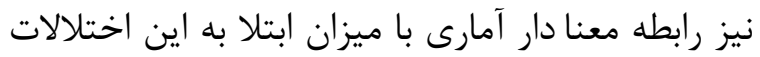

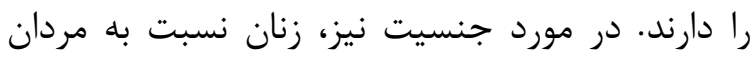

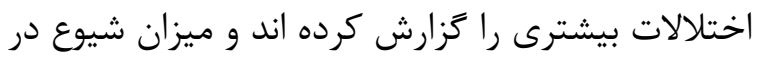

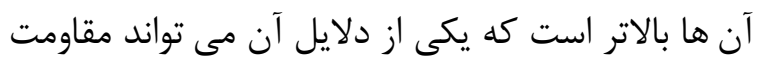

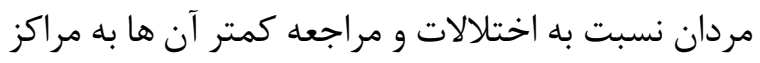

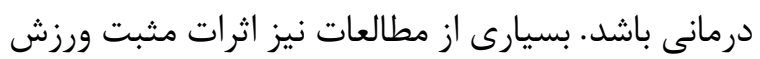
بر كاهش علائم MSDs، و به ويز مطئه در اندام هاى فوقانى 
تعدادى از شعب (خوشه) به صورت تصادفى انتخاب شده

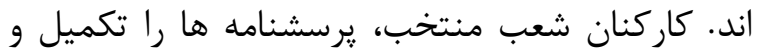

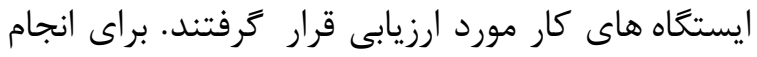

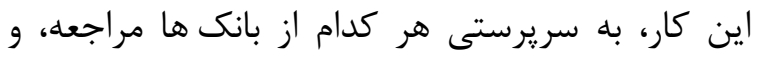

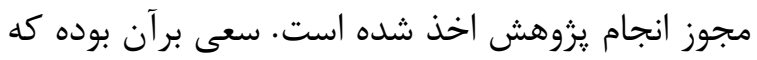

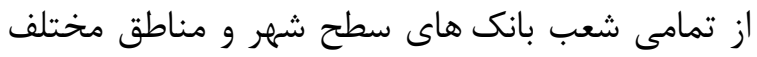

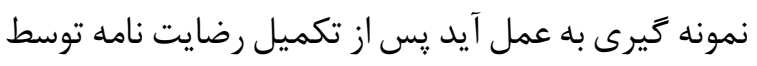

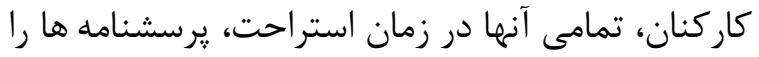

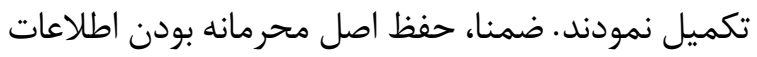

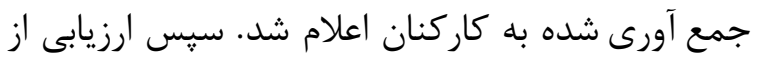

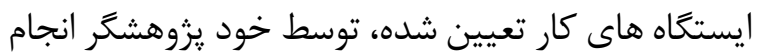

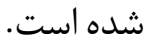

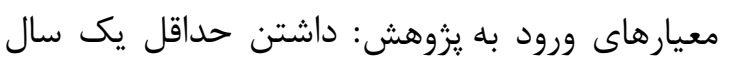

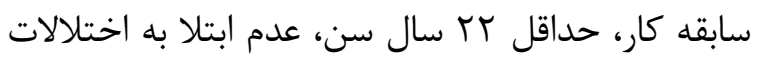

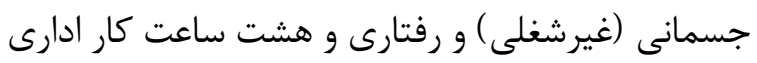

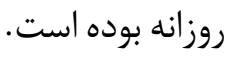

ب) شيوه جمعآورى داده:

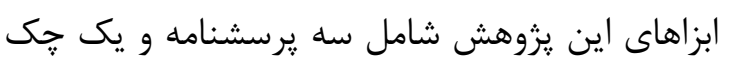

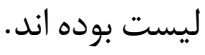

\section{بخش اول: برسشنامه دموكر/فيك:}

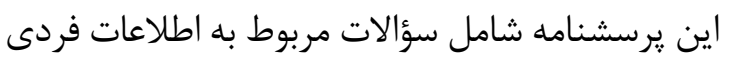

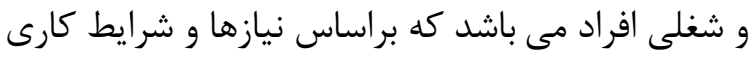

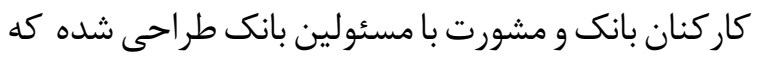

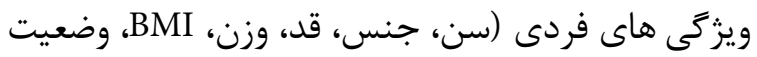

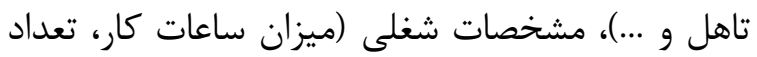

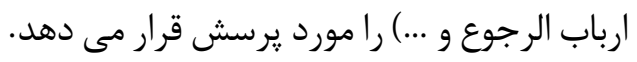

بخش دوم: جك ليست /رزيابى /ركونوميكى محيط كار

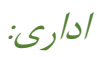

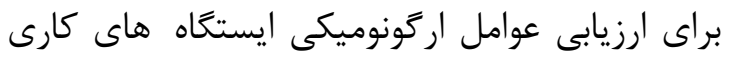

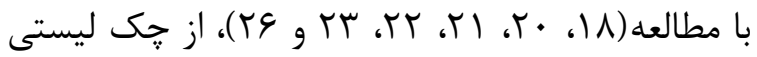

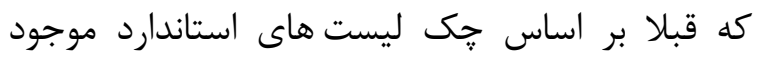

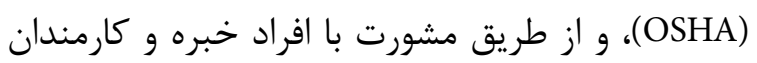

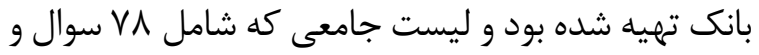

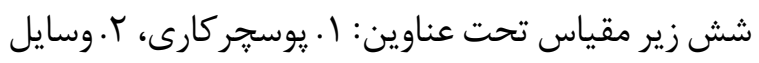

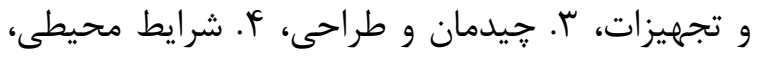

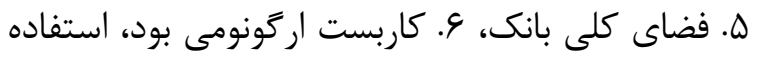

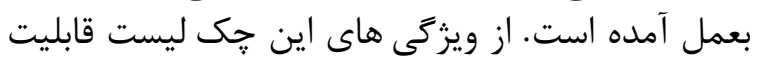

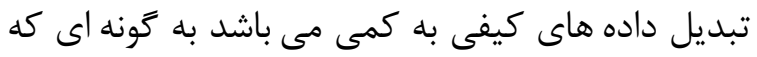

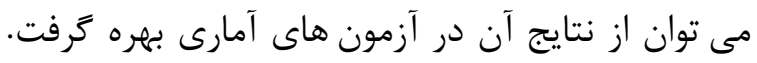

داشت، نمره كيفيت زندگى كاركنان به طور معنادارى

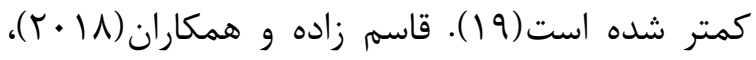

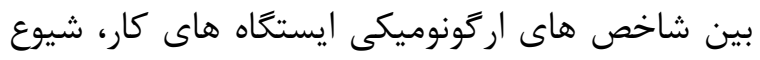

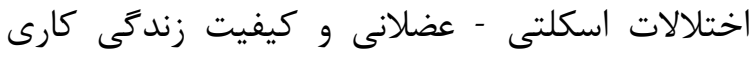

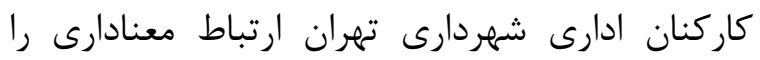

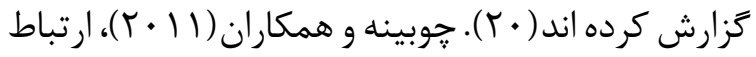

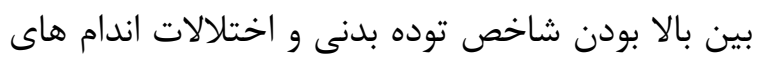

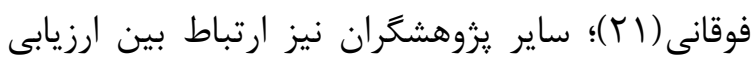

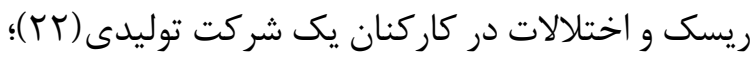

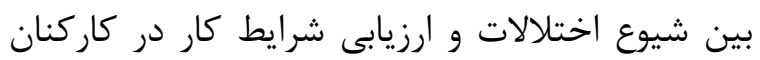

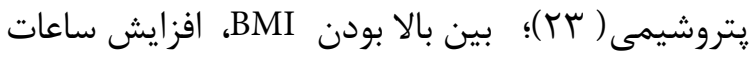

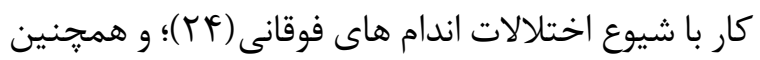

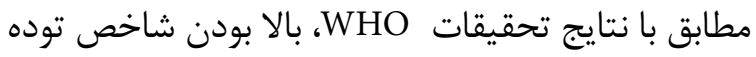

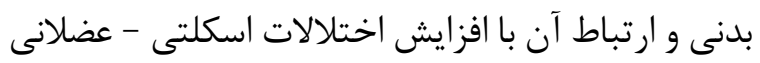

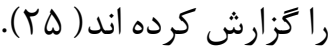

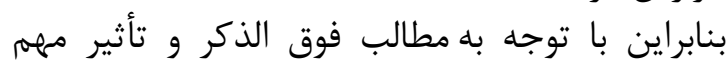

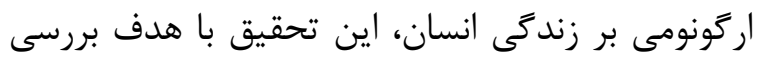

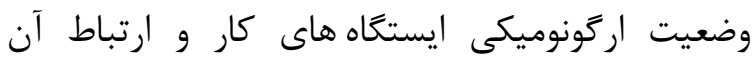

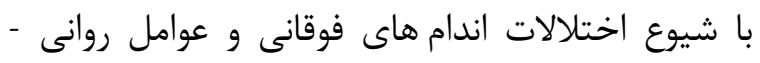

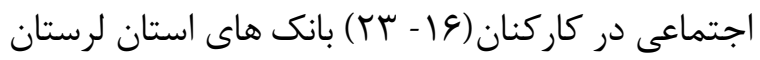

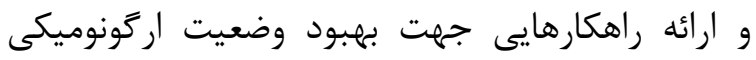

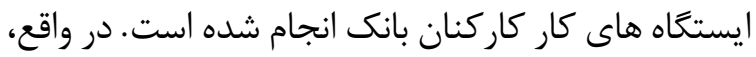

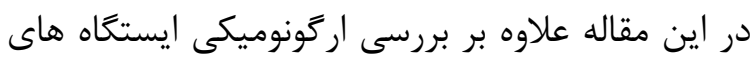

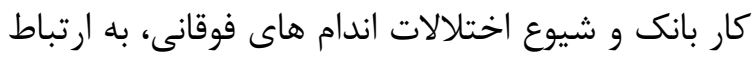

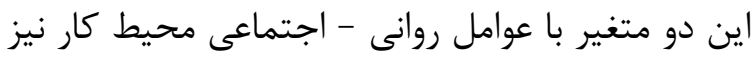

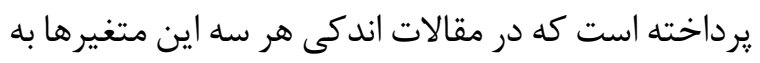

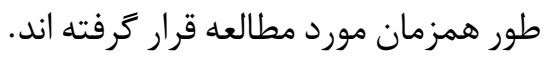

روش بررسى يزوهش حاضر بر اساس هدف كاربردى و بر اساس نحوه

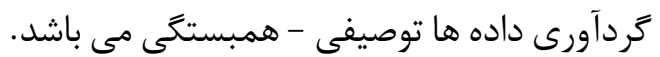

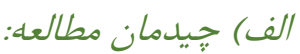

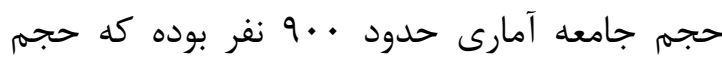

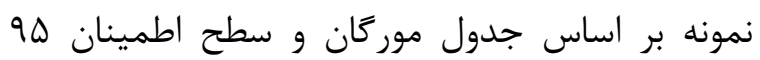

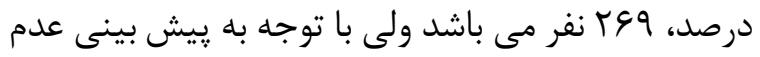

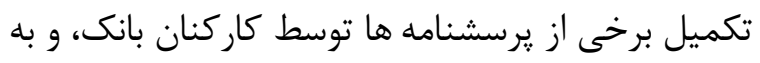

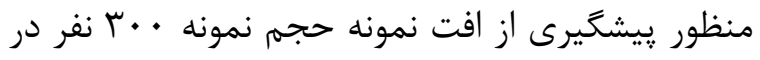

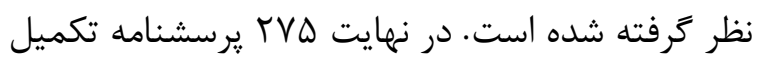

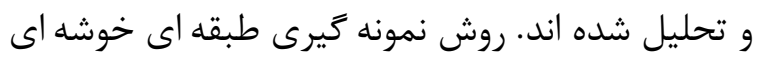

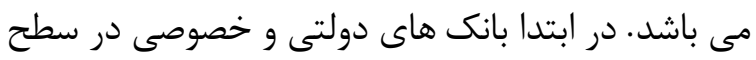

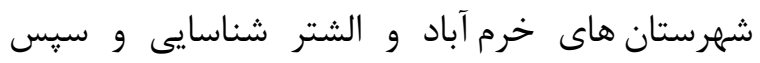


بخش جهارم: برسشنامه عوامل روانى - اجتماعى:

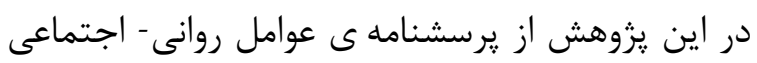

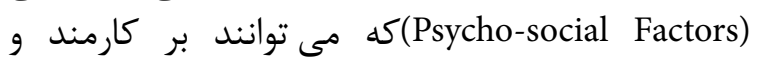

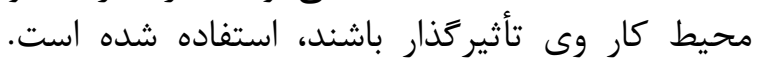

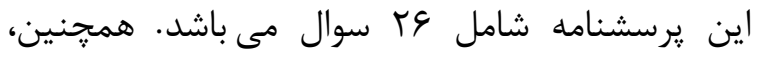

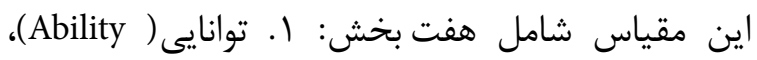

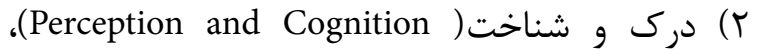

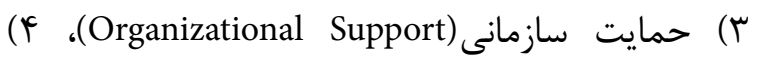

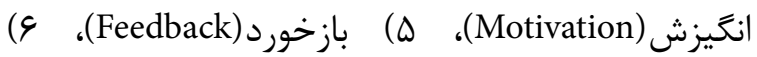

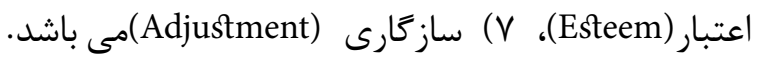

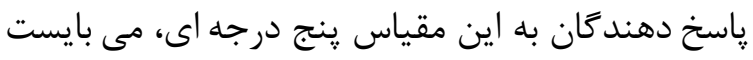

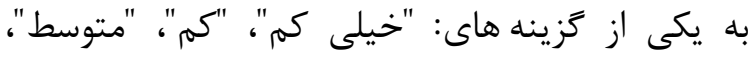

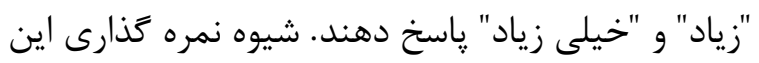

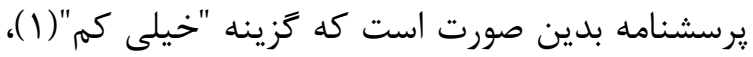

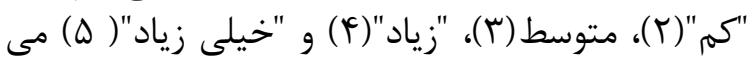

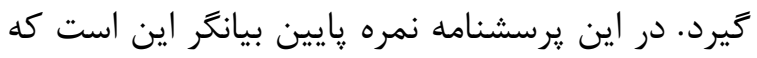

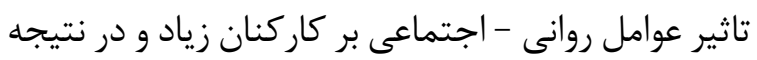

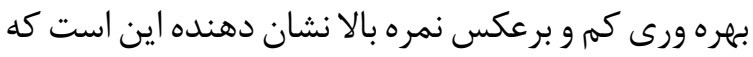

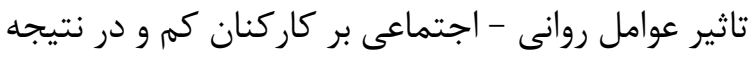

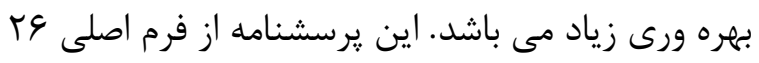

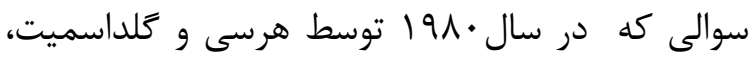
تهيه شده، بركرفته شده

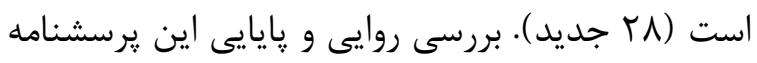

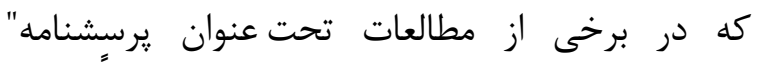

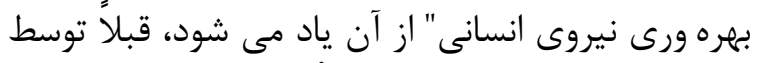

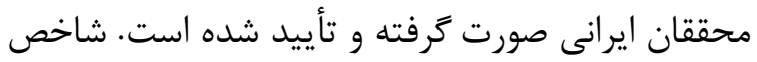

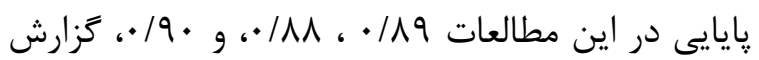

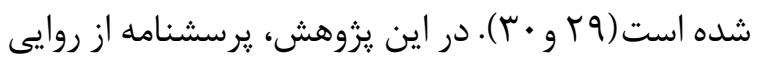

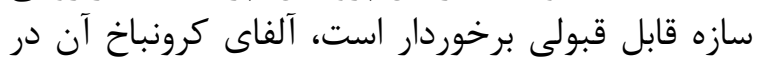

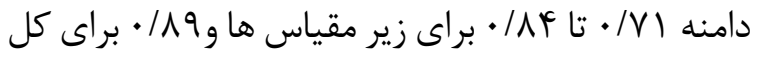

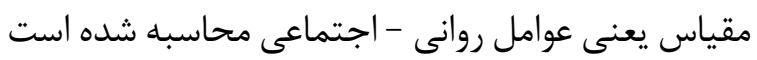

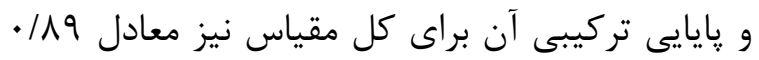

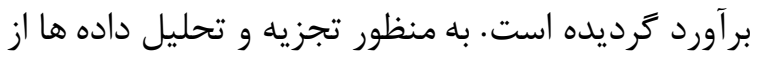

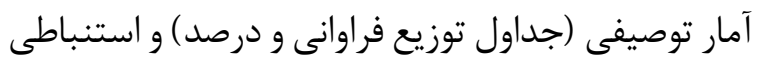

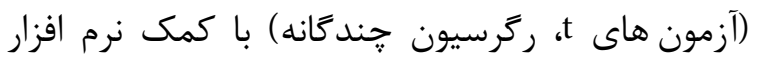
SPSS 23 SPاستفاده به عمل آمده.

ج) تتجزيه و تحليل آمارى داده ها:

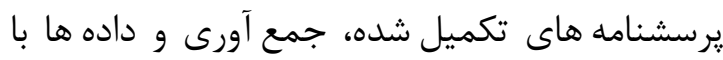

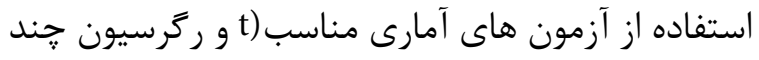

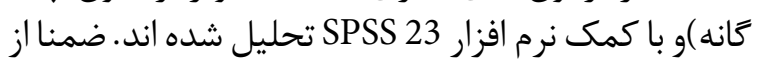

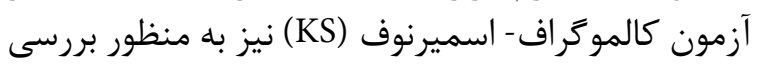

سؤالات جك ليست به صورت "بلى"، ، "خير" و يا "كاربرد

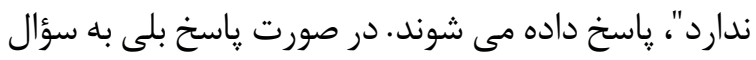

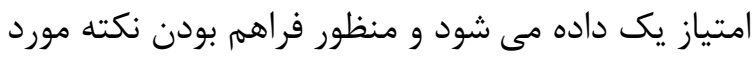

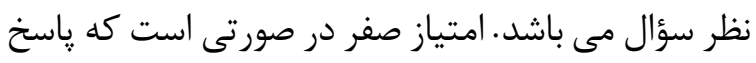

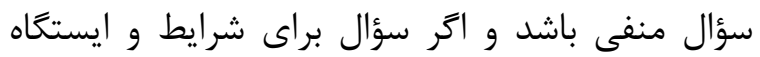

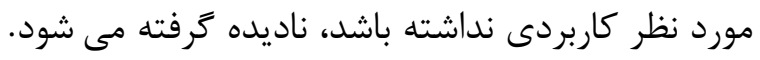

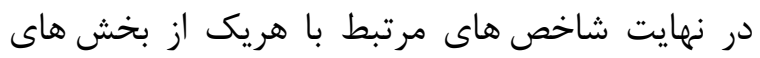

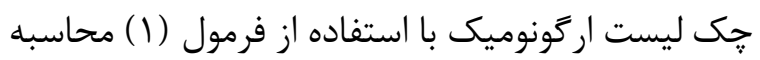

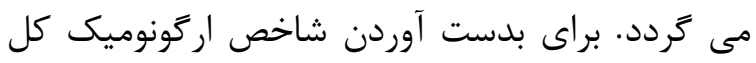

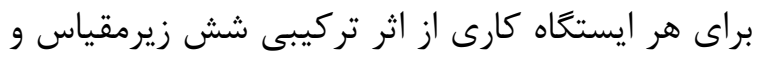

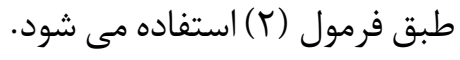

$$
E i=x i \times 100 / n i-l i
$$

در فرمول فوق Ei شاخص بخش i ام جك ليست،

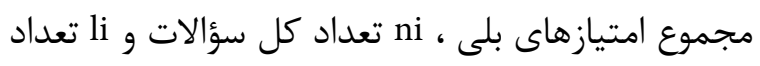

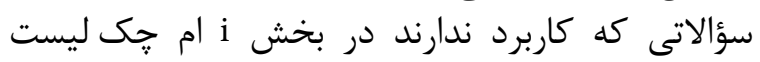

$$
T=\sum_{i=1}^{5} x \times 100 / N-L
$$

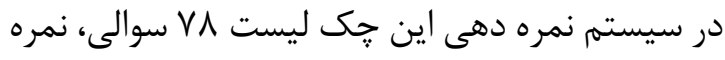

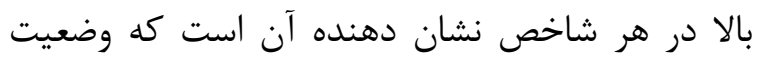

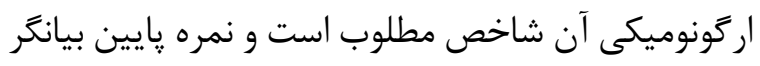

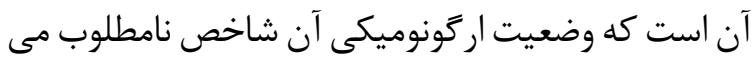

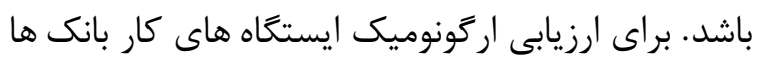

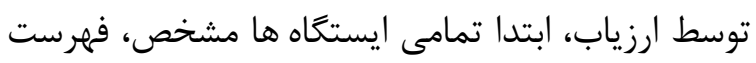

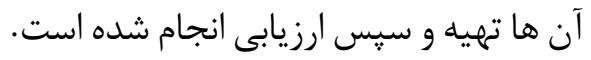

بخش سوم: برسشنامه اختلالات اسكلتى-عضلانى

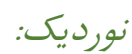
در اين روش با استفاده از يرسشنامه هايى كه

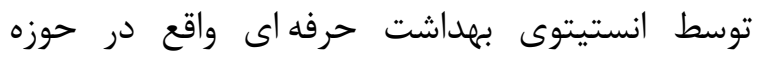

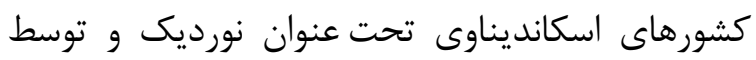

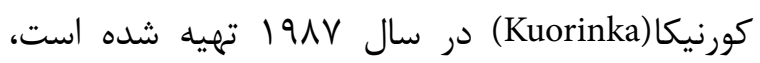

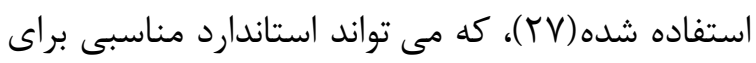

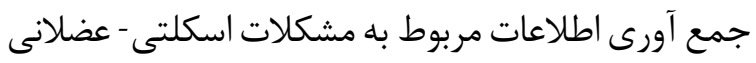

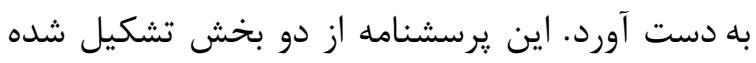

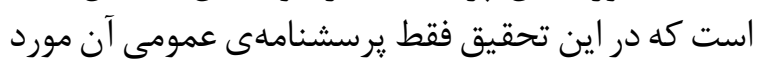

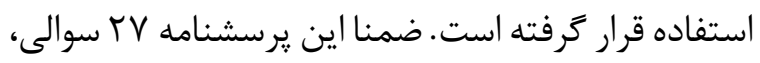

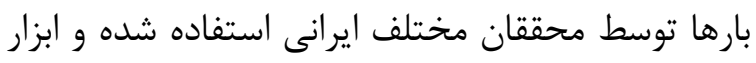

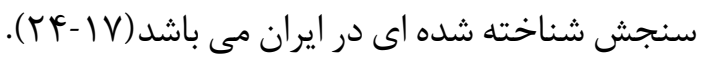


ارگَونومى" مى باشد. اين به آن معناست كه در اقدامات

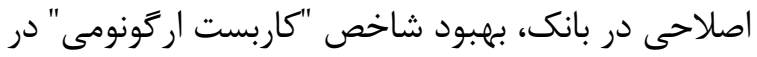
اولويت قرار دارد.

با توجه به جدول بّ سو با تأكيد بر مقادير t به دست آمده،

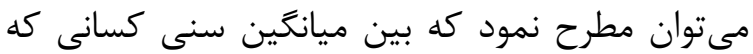

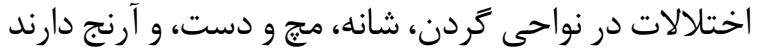

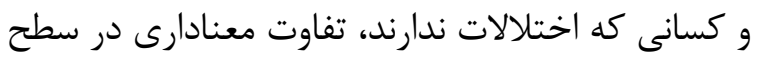

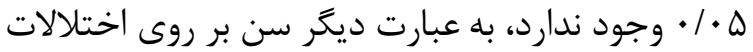

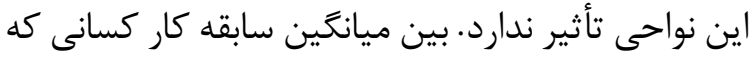

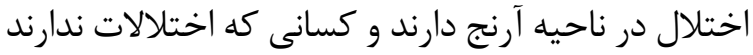

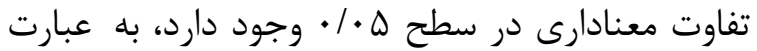

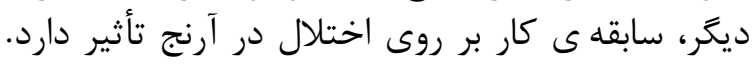

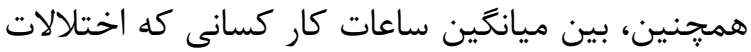

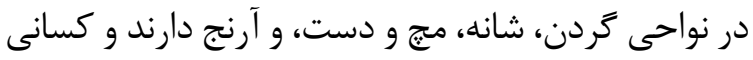

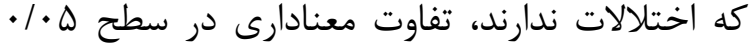

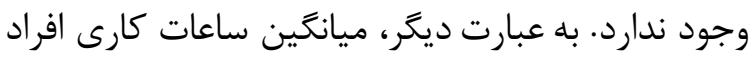

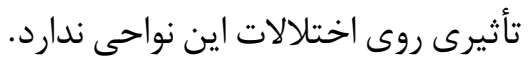

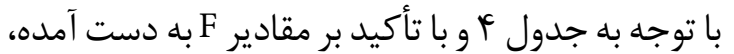

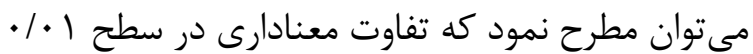

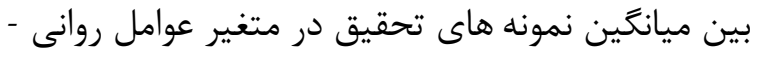

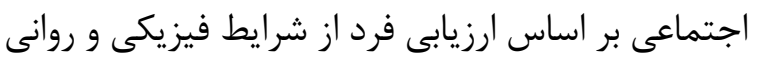
محيط كار خود، وجود دارد. (شايان ذكر است است درئ در اين

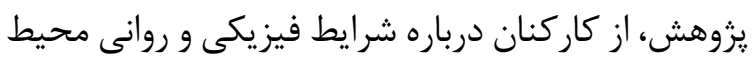

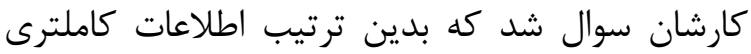

$$
\text { بدست آورده شود). }
$$

نرمال بودن داده ها استفاده شده كه فرضيه نرمال بودن

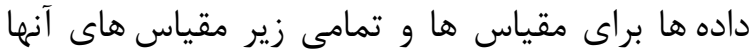
برقرار مى باشد. (p>05/0)

يافته ها

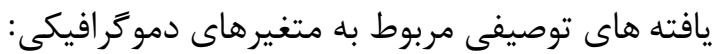

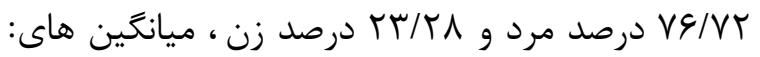

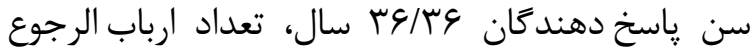

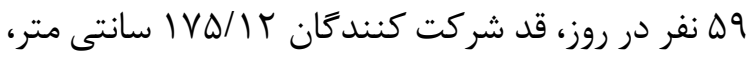

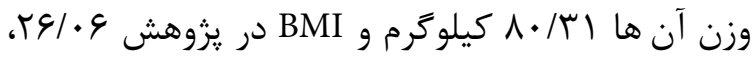

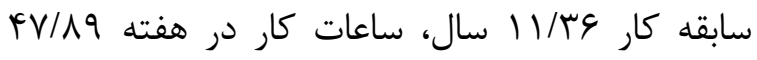

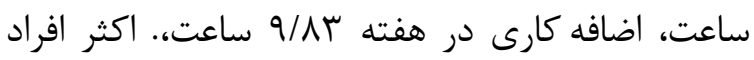

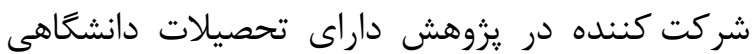

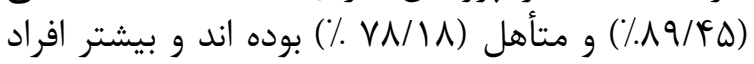

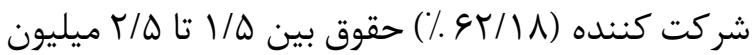
تومان دريافت مى كنيند

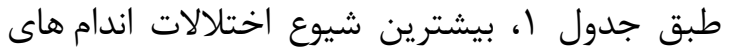

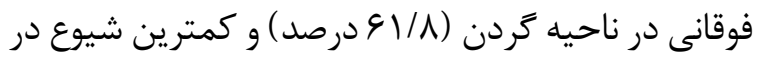

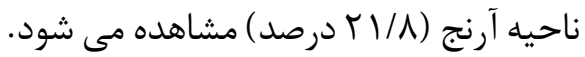

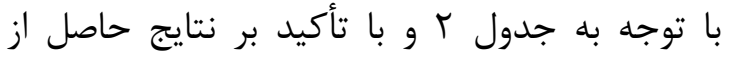

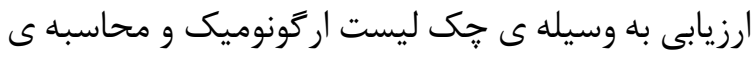

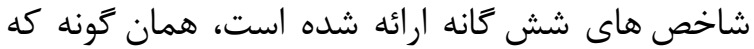

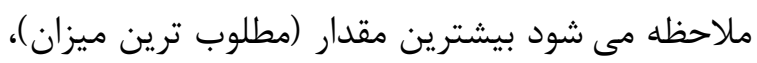

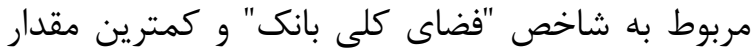

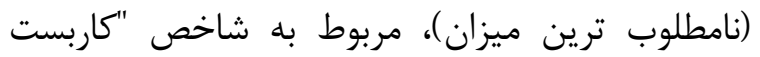

جدول ا. توزيع فراونى شيوع اختلالات اندام هاى فوقانى

\begin{tabular}{|c|c|c|}
\hline \multicolumn{2}{|c|}{ فراوانى (درصد) } & \multirow{2}{*}{ ناحيه } \\
\hline بدون اختلال & داراى اختلال & \\
\hline $1.9(\Gamma \Lambda \% / \Gamma)$ & $199(91 \% / 1)$ & تحردن \\
\hline $\operatorname{ITV}(\leftarrow \& \mid \%)$ & $\operatorname{IF\wedge }(\Delta \mu / \%$ ( & 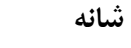 \\
\hline $\operatorname{lQT}(\Delta \Delta \mid \% \cdot \%)$ & $\operatorname{IrT}\left(F \kappa \% \% T^{\kappa}\right)$ & مج و دست \\
\hline rID(YN/\% $\%$ ) & S.(T) $(Y \% . \wedge)$ & آرنج \\
\hline
\end{tabular}

جدول r. ميانگين و انحراف استاندارد شاخص هاى ارگونوميك در ايستخاه كار

\begin{tabular}{|c|c|c|c|c|}
\hline حداكثر & 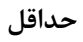 & انحراف استاندارد & ميانغين & 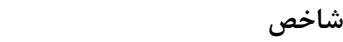 \\
\hline $9 \mathrm{~V} / .9$ & $11 / 94$ & $M T / Q$. & $9 K / 94$ & شاخص يوسجر كارى \\
\hline Q९/VT & F/VT & $r F / l$. & $r q / \mu r$ & شاخص وسايل و تجهيزات \\
\hline $9 \Delta / T V$ & $9 / 9$. & $r T / \pi$. & $9 \Delta / 9 \Lambda$ & شاخص جيدمان و طراحى \\
\hline$v * / 9$. & $r / \mu q$ & $r \Delta / v \wedge$ & rN/.r & شاخص شرايط محيطى \\
\hline IV/A) & FN/VT & $14 / 19$ & $\Lambda \cdot / \kappa \cdot$ & شاخص فضاى كلى بانك \\
\hline $4 \cdot \pi q$ & $1 / \cdot 9$ & $\mid r / r \Lambda$ & N/VT & شاخص كاربست اركونومى \\
\hline IV/AI & $1 / \cdot 9$ & $r$ r/qQ & $\Delta \varphi / V$. & شاخص كل اركونوميكى ايستكاه كار \\
\hline
\end{tabular}


شهناز طباطبايى و مصطفى حسنوند

جدول س. تفاوت ميانگين در اختلالات اندام هاى فوقانى با ملاحظه بر متغيرهاى "اسن، سابقه كار و ساعات كاره آزمون t دو كروه مستقل

\begin{tabular}{|c|c|c|c|c|c|c|c|}
\hline Sig & Df & $\mathbf{T}$ & SD & ميانكين & تعداد & اختلال & متغير \\
\hline & & & & سن & & & \\
\hline \multirow{2}{*}{.1 .99} & \multirow{2}{*}{ TVT } & \multirow{2}{*}{ l/ATV } & GITIV & $r \Delta / \mathcal{E V}$ & $1 \cdot 0$ & بدون & \multirow{2}{*}{ كردن } \\
\hline & & & GIVFT & $r 9 / 90$ & 199 & داراى & \\
\hline \multirow{2}{*}{$\cdot / r \cdot$} & \multirow{2}{*}{ TVR } & \multirow{2}{*}{. /Arq } & q/9.r & Tr/ & ITV & بدون & \multirow{2}{*}{ شانه } \\
\hline & & & ध/रqा & $r G / 4$. & $1 F \lambda$ & داراى & \\
\hline \multirow{2}{*}{ • /TVK } & \multirow{2}{*}{ TVR } & \multirow{2}{*}{$\cdot / r T \Lambda$} & 9190 & rזוצr & IQT & بدون & \multirow{2}{*}{ مج و دست } \\
\hline & & & $g / 4 \lambda$ & rG/KG & ITr & داراى & \\
\hline \multirow{3}{*}{$\cdot \pi \cdot 4$} & \multirow{2}{*}{ TVR } & \multirow{2}{*}{$\cdot / 9 \cdot V$} & $g / 4 \Lambda$ & $r \xi / \Delta \Delta$ & rIQ & بدون & \multirow{2}{*}{ آرنج } \\
\hline & & & $9 / 1 \Lambda$ & $r \Delta /{ }^{\prime} \Lambda$ & 4. & داراى & \\
\hline & & & & سابقه كار & & & \\
\hline \multirow{2}{*}{$\cdot / \cdot \wedge \Lambda$} & \multirow{2}{*}{ TVR } & \multirow{2}{*}{ I/VIT } & $V / \cdot \Delta r$ & $1 \cdot|4|$ & $1 \cdot \Delta$ & بدون & \multirow{2}{*}{ كردن } \\
\hline & & & $V / r \cdot r$ & $11 / 94$ & IV. & داراى & \\
\hline \multirow{2}{*}{$\cdot / r \Delta q$} & \multirow{2}{*}{ TVR } & \multirow{2}{*}{$1 / 1 \Delta \Delta$} & $V|\Delta \varphi|$ & $11 / r \wedge$ & ITV & بدون & \multirow{2}{*}{ شانه } \\
\hline & & & $9 / 990$ & $11 / F r$ & 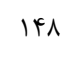 & داراى & \\
\hline \multirow{2}{*}{. } & \multirow{2}{*}{ TVT } & \multirow{2}{*}{ • } & $1 / 4 V$ & $T / V I$ & IQT & بدون & \multirow{2}{*}{ مج و دست } \\
\hline & & & $|/ 4|$ & $r / 9 \Delta$ & ITT & داراى & \\
\hline \multirow{3}{*}{$\cdot 1 \cdot r q$} & \multirow{2}{*}{ TVR } & \multirow{2}{*}{$-r / \cdot 9$} & $1 / r \Lambda$ & $r / 9 \Lambda$ & rID & بدون & آرنح \\
\hline & & & $9 \Delta 1$ & $r / V$ & c. & داراى & اربج \\
\hline & & & & ساعات كار & & & \\
\hline .1 .90 & raT & 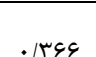 & $\Lambda / r \Delta q$ & $F q / v \Delta$ & $1 . r$ & بدون & \\
\hline & & . N>Y & $1 \cdot / r \Delta q$ & $q q / r$. & 194 & داراى & كردن \\
\hline .1 .90 & r & I/rur & 9/9YI & $\Delta \cdot / r \mid$ & $|r|$ & بدون & لح شانه \\
\hline $.1 \cdot 7 \omega$ & 191 & WAN & $9 / 195$ & $|F| \wedge \mid$ & lff & داراى & ساهل \\
\hline.$\pi \cdot \varphi$ & שע &.$- / 1 \Delta f$ & $r / T$ & FV/AD & $1 \& \lambda$ & بدون & مح مدנست \\
\hline$\cdot \pi \cdot 7$ & TrT & $-\cdot / Q T t$ & g/4G & FV/qA & $11 V$ & داراى & مج و دس \\
\hline.$/ 4 q$. & T T T & I/FTF & $V / .9$ & FN/TH & $r \cdot V$ & بدون & آرنج \\
\hline 政1 & 开 & 1/111 & 91.9 & fe/vq & $\Delta \Lambda$ & داراى & ارتج \\
\hline
\end{tabular}

با توجه به جدول \& و با تأكيد بر ميزان F به دست آمده،

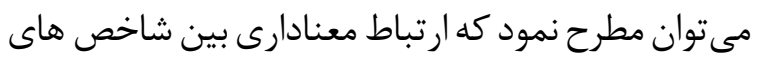

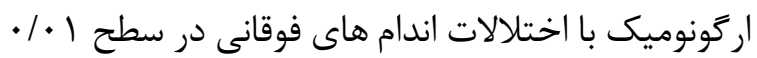

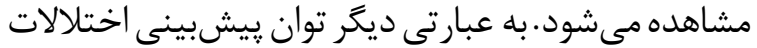

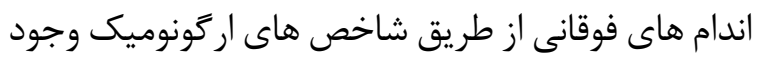

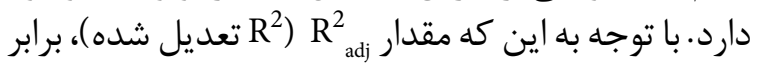

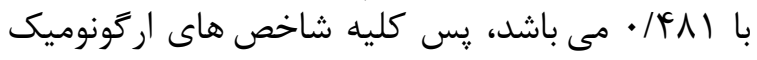
به جز شاخص شرايط محيطى در اين مدل بـ بأ درصد

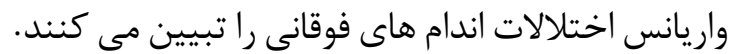

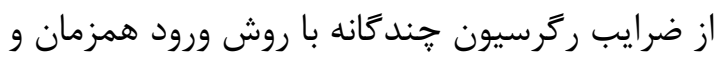

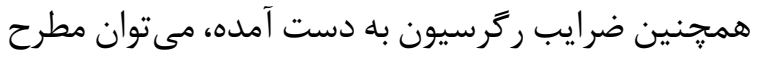

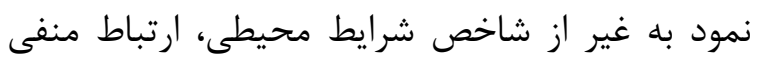

با توجه به جدول له نتيجه گرفته مى شود كه به جز شاخص شرايط محيطى، بين شاخص هاى يوسجر كارى،

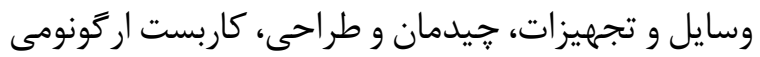

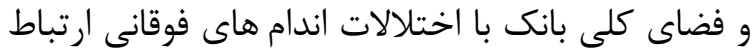
معكوس و معنادارى وجود دارد، و به طور كلى مى توان

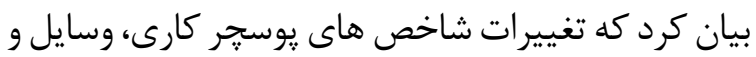

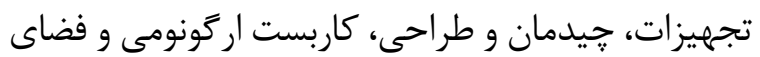
كلى بانكى با اختلالات اندام هاى فوقانى دئى در آزمودنى هاتى هاى تحقيق همعام و معكوس بوده و افزايش متغير اول (يعنى

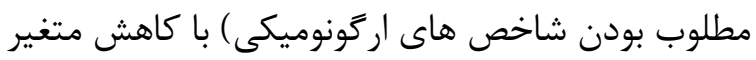
ديخر (يعنى كاهش اختلالات اندام هاى فوقانى) همراه 
بر رسى ارگونوميك ايستخاه هاى كار ...

جدول F. تحليل واريانس يك طرفه جهت بررسى "عوامل روانى- اجتماعى" با ملاحظه بر "ارزيابى فرد از شرايط فيزيكى و روانى محيط كار خود"

\begin{tabular}{|c|c|c|c|c|c|c|}
\hline Sig & df & $\mathbf{F}$ & SD & ميانكَين & & متغير \\
\hline \multicolumn{7}{|c|}{ ارزيابى فرد از شرايط فيزيكى محيط كار } \\
\hline \multirow{5}{*}{$\cdot \cdots$} & \multirow{5}{*}{ r } & \multirow{5}{*}{$\Delta / T \Delta F$} & - IGFF & $r / r I$ & عالى & \multirow{5}{*}{ عوامل روانى } \\
\hline & & & . $19 \Delta$ & $r / l r$ & 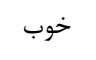 & \\
\hline & & & $\cdot / \Delta T$. & $r / 9 q$ & 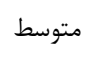 & \\
\hline & & & $\cdot \mid \Delta \wedge \phi$ & r/A & 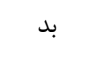 & \\
\hline & & & .1999 & t/M & 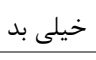 & \\
\hline \multicolumn{7}{|c|}{ ارزيابى فرد از شرايط روانى محيط كار } \\
\hline \multirow{5}{*}{$\cdot / \cdot v$} & \multirow{5}{*}{ f } & \multirow{5}{*}{ T/DGT } & $\cdot 1999$ & T/AF & 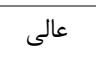 & \multirow{5}{*}{ عوامل روانى } \\
\hline & & & . & $r / T F$ & خوب & \\
\hline & & & $\cdot / \Delta T \Lambda$ & r & 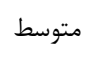 & \\
\hline & & &.$|9|$. & r/q. & بد & \\
\hline & & & $.19 \cdot 4$ & T/AF & خيلى بد & \\
\hline
\end{tabular}

جدول ه. ماتريس همبستَى يِيرسون بين "شاخص هاى اركونوميك كل" و 》اختلالات اندام هاى فوقانى"

\begin{tabular}{|c|c|c|}
\hline سطح معنادارى & همبستخى & متغيرها \\
\hline $.1 \cdots$ & $-\cdot|\Delta \Lambda|$ & يوسجر كارى / اختلالات اندام هاى فوقانى \\
\hline.$/ \cdot 4$ & $-\cdot / r /$ & وسايل و تجهيزات / اختلالات اندام هاى فوقانى \\
\hline. & $-\cdot A T V$ & جيدمان و طراحى / اختلالات اندام هاى فوقانى \\
\hline$\cdot / 1 \cdot r$ & $-\cdot / \cdot V V$ & شرايط محيطى / اختلالات اندام هاى فوقانى \\
\hline $.1 \cdot r \Delta$ & 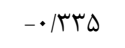 & كاربست اركونومى / اختلالات اندام هاى فوقانى \\
\hline.$\mu r$ & -.109. & فضاى كلى / اختلالات اندام هاى فوقانى \\
\hline
\end{tabular}

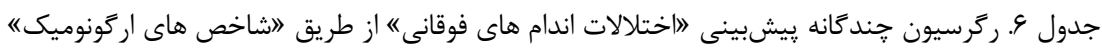

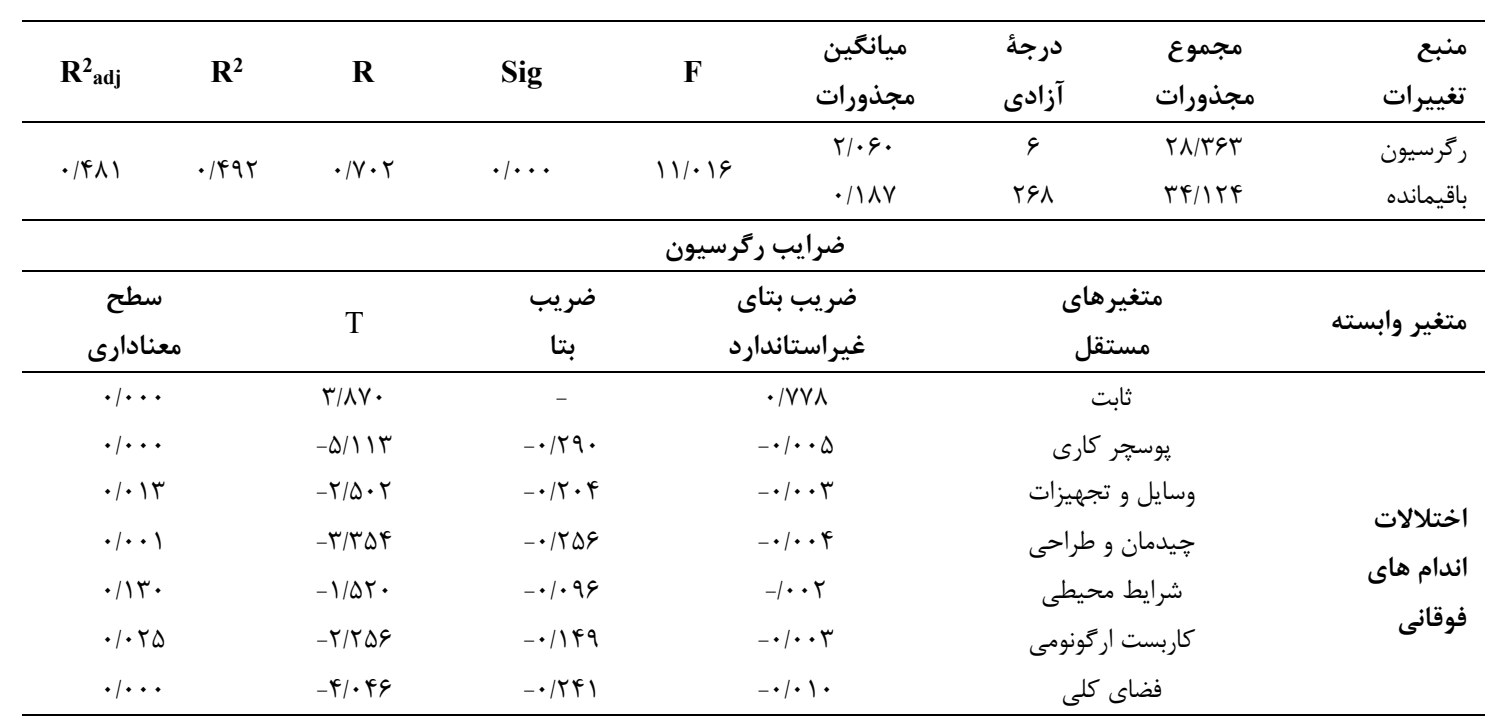


جدول V. ماتريس همبستخى ييرسون بين "عوامل روانى - اجتماعى" و 》اختلالات اندام هاى فوقانى"

\begin{tabular}{|c|c|c|}
\hline سطح معنادارى & همبستكى & متغيرها \\
\hline .1499 & $-\cdot / \cdot F f$ & توانايى / اختلالات اندام هاى فوقانى \\
\hline$\cdot / \cdot 11$ & $-\cdot / l \Delta \mu$ & درى و شناخت / اختلالات اندام هاى فوقانى \\
\hline.$/ \cdot 9$ & $-\cdot / l \Delta V$ & حمايت سازمانى / اختلالات اندام هاى فوقانى \\
\hline . & -0 & انكَيزش / اختلالات اندام هاى فوقانى \\
\hline $.1 \cdot 11$ & $-\cdot / l \Delta F$ & بازخورد / اختلالات اندام هاى فوقانى \\
\hline$\cdot / \cdots$ & $-\cdot|r| \cdot$ & اعتبار / اختلالات اندام هاى فوقانى \\
\hline.$/ .1 \mathrm{~V}$ &.$- / l k r$ & سازحارى/ اختلالات اندام هاى فوقانى \\
\hline
\end{tabular}

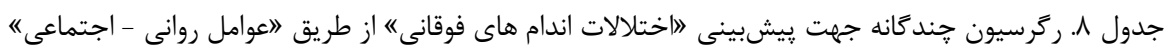

\begin{tabular}{|c|c|c|c|c|c|c|c|c|}
\hline $\mathbf{R}^{2}{ }_{\text {adj }}$ & $\mathbf{R}^{2}$ & $\mathbf{R}$ & Sig & $\mathbf{F}$ & ميانكين مجذورات & درجه آزادى & مجموع مجذورات & منبع تغييرات \\
\hline \multirow{2}{*}{. } & \multirow{2}{*}{ ( } & \multirow{2}{*}{$\cdot \mid \Delta \Lambda$} & \multirow{2}{*}{$\cdot 1 \cdot \cdot 1$} & \multirow{2}{*}{$1 T / 9 \cdot 9$} & $\mid H / H Q V$ & v & TQ/ATA & ركرسيون \\
\hline & & & & & $\cdot / T \wedge V$ & reV & FI/GTT & باقيمانده \\
\hline \multicolumn{9}{|c|}{ ضرايب رَرسيون } \\
\hline Sig & \multicolumn{2}{|l|}{$\mathrm{T}$} & ضريب بتا & & ضريب بتاى غير استاندارد & \multicolumn{2}{|c|}{ متغيرهاى مستقل } & متغير وابسته \\
\hline$\cdot 1 \cdots$ & \multicolumn{2}{|c|}{$-1 \cdot / 491$} & - & & 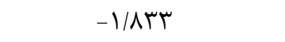 & \multicolumn{2}{|c|}{ ثابت } & \\
\hline - /AMF & \multicolumn{2}{|c|}{$-\cdot|\pi|$} & -.1 .19 & &.$- / \cdot 14$ & \multicolumn{2}{|c|}{ ت انايى } & \\
\hline$\cdot 1 \cdots$ & \multicolumn{2}{|c|}{ hatet } & $-\cdot / I V F$ & & $-\cdot / I V K$ & \multicolumn{2}{|c|}{ درك و شناخت } & \\
\hline$\cdot \cdot v$ & \multicolumn{2}{|c|}{$-r / V I V$} & $-\cdot / 1 \wedge \Delta$ & & $-\cdot 11 \cdot 1$ & \multicolumn{2}{|c|}{ حمايت سازمانى } & \\
\hline - $/ \lambda \mu F$ & \multicolumn{2}{|c|}{$-\cdot|r|$} &.$- \cdot 1 \cdot 19$ & &.$- \cdot \cdot 1 r$ & \multicolumn{2}{|c|}{ انغيزش } & اختلالات اندام ه \\
\hline$\cdot / \Lambda 9 V$ & \multicolumn{2}{|c|}{$-\cdot / 19 V$} &.$- .1 \cdot 1 r$ & & $-.1 . .99$ & \multicolumn{2}{|c|}{ بازخورد } & \\
\hline $.1 \cdot 9$ & \multicolumn{2}{|c|}{$-r / \cdot \cdot v$} & $-\cdot|1 \wedge|$ & & -.1 .90 & \multicolumn{2}{|c|}{ اعتبار } & \\
\hline$\cdot / \cdot r$. & \multicolumn{2}{|c|}{$-r / I V V$} & $-\cdot / 1 r 4$ & & $-\cdot / \cdot v \cdot$ & \multicolumn{2}{|c|}{ سازگًارى } & \\
\hline
\end{tabular}

فوقانى) همراه است.

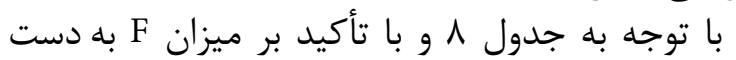

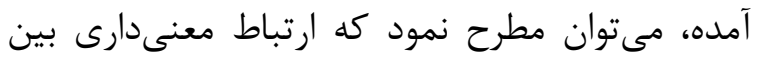

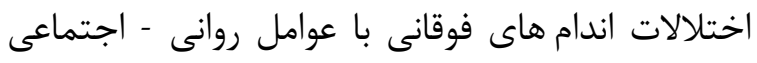

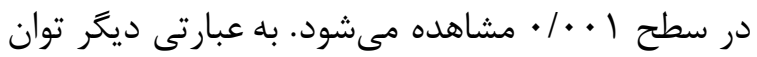

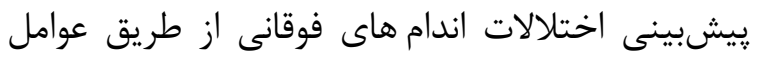

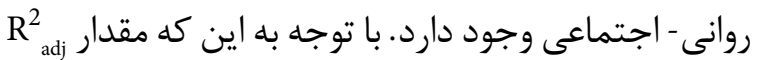

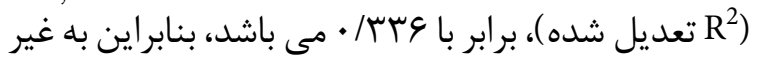

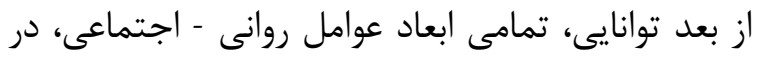

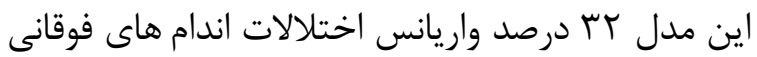

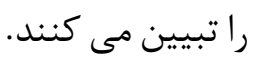

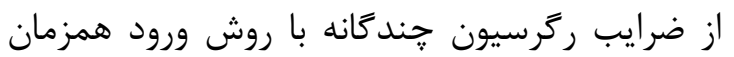

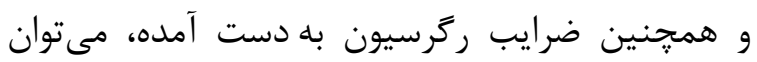

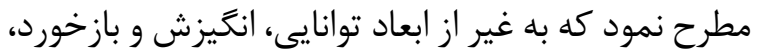

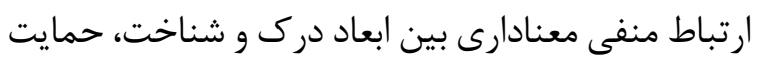

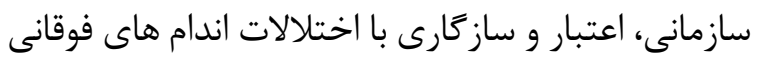

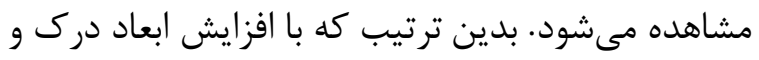

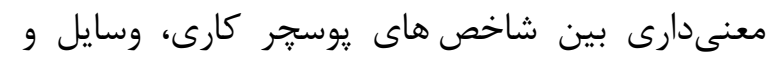

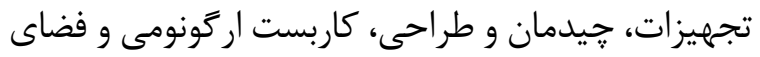

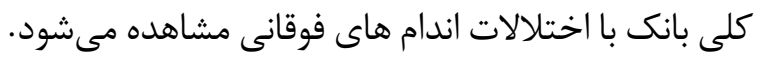

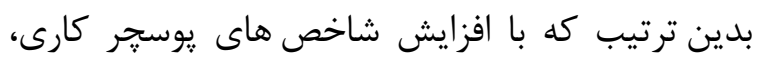

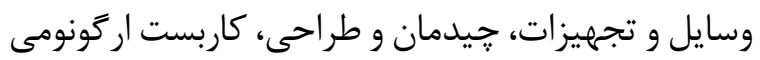

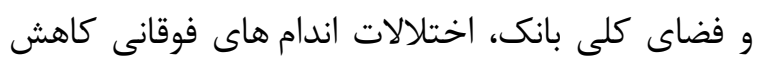

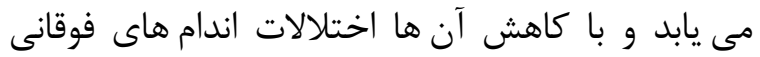
افزايش مى يايد.

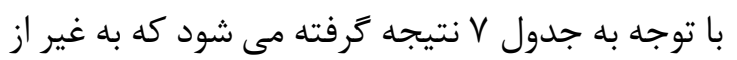

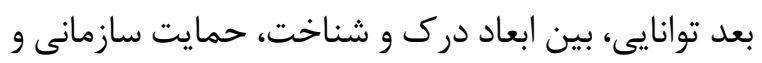

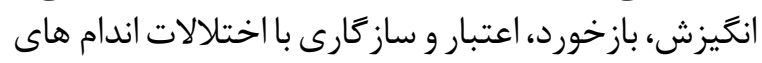

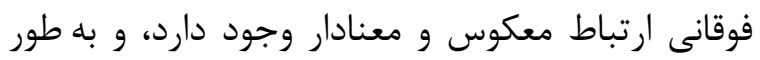

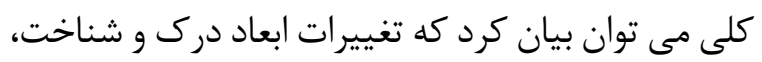

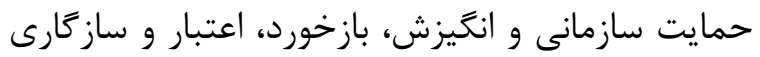

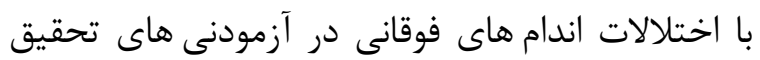

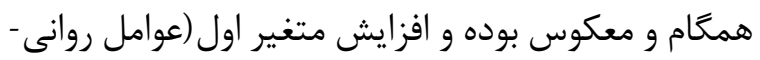
اجتماعى) با كاهش متغير ديخر (اختلالات اندام هاى 
ارتباط معنادار وجود دارد(ه، 9 و • · (Y). در تحقيق

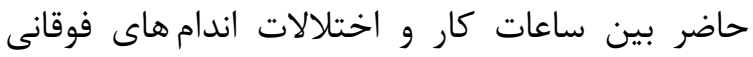

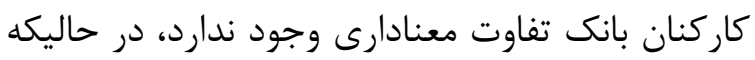

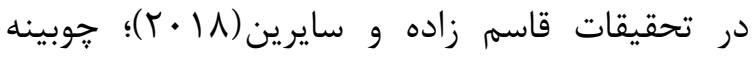

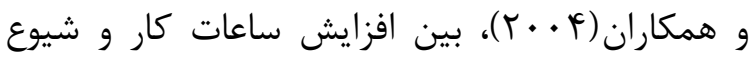

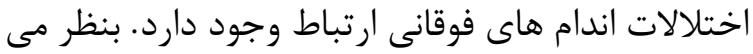

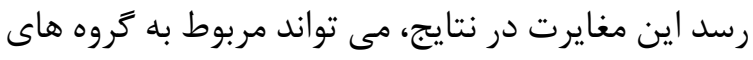

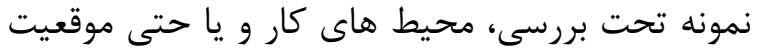

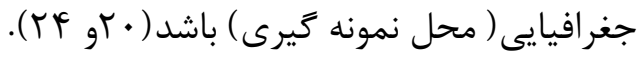

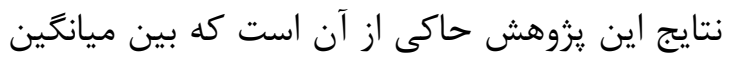

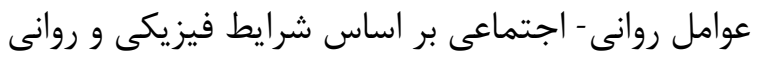

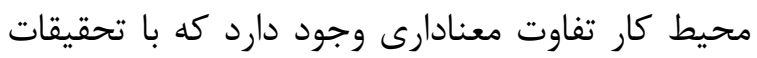

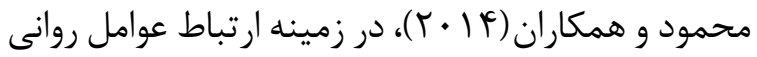

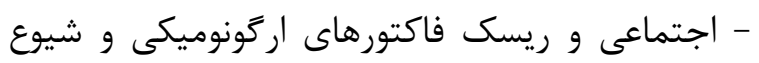

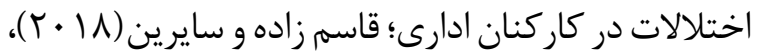

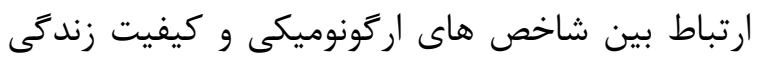

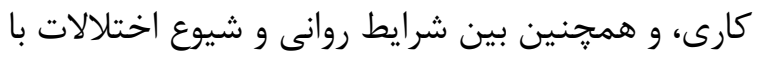

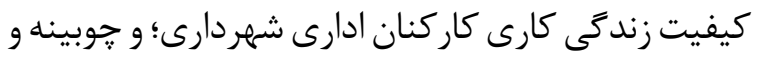

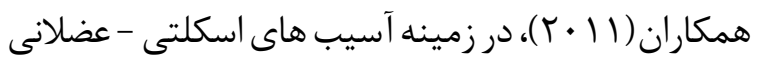

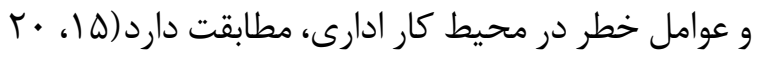

به طور كلى مى توان نتيجه كيرى كرد كه عوامل روانى كإنى

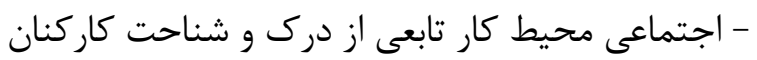

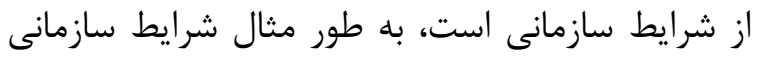

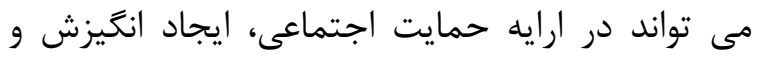

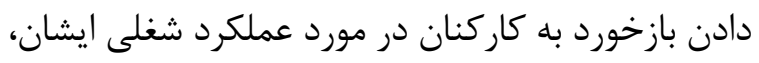

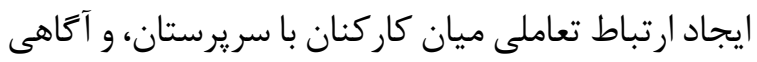

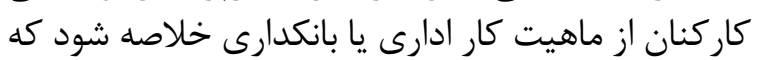

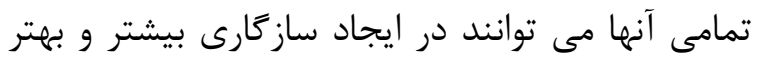

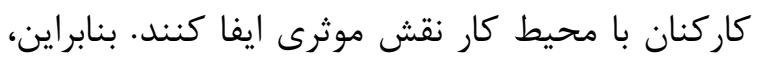

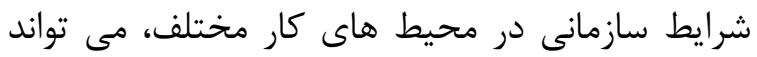

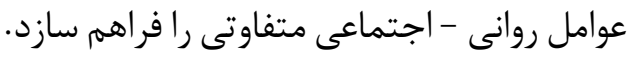

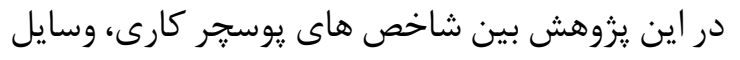

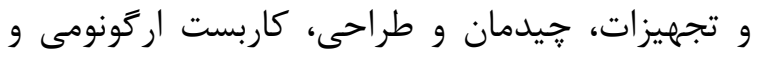

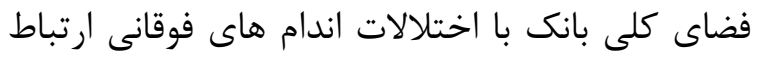

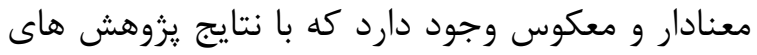

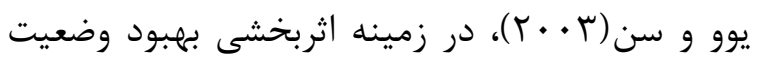

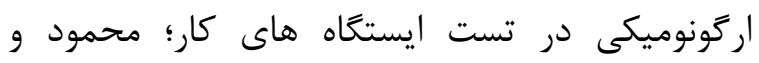

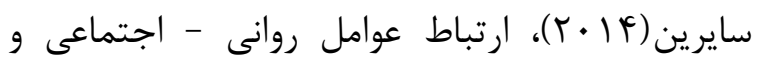

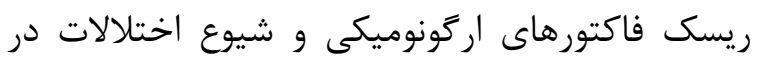

شناخت، حمايت سازمانى، اعتبار و سازكارى، اختلالات

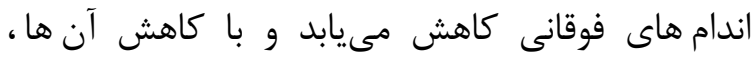
اختلالات اندام هاى فوقانى افزايش ميى فيابد.

بحث نتايج حاصل از اين يزوهش نشان مى دهد كه بيشترين

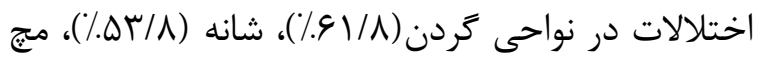

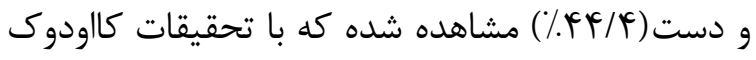

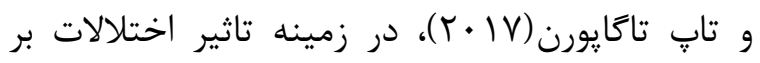

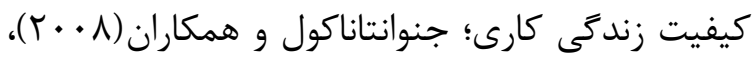

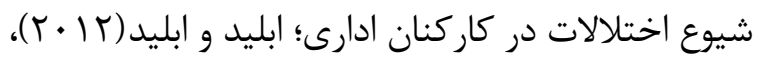

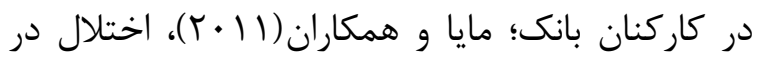

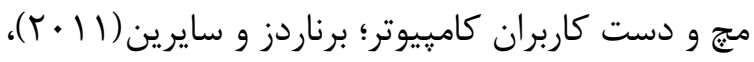

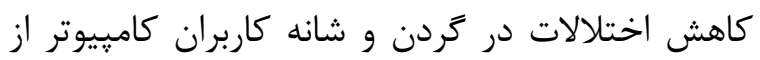

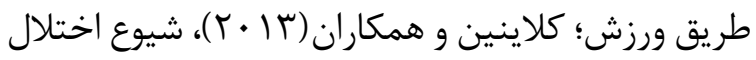

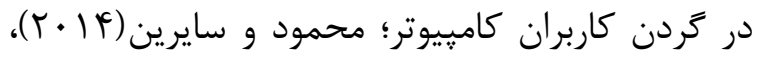

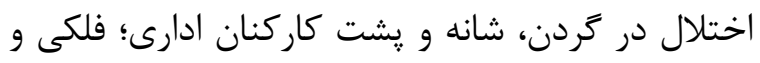

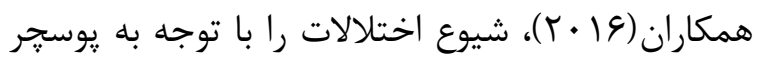

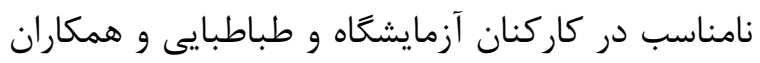

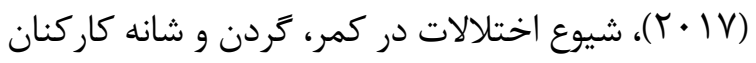

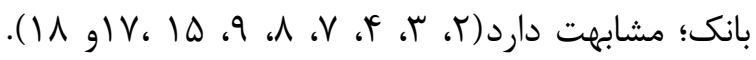

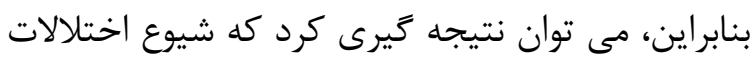

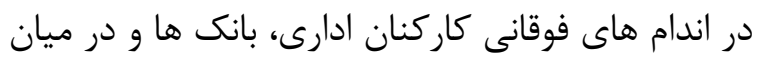
كاربران كامييوتر بيشتر است كان.

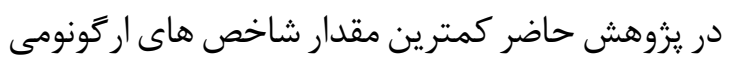

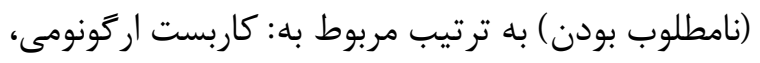

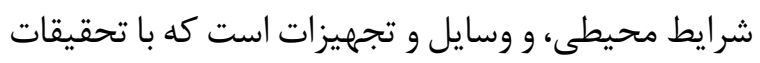

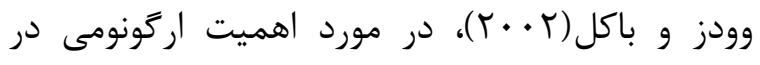

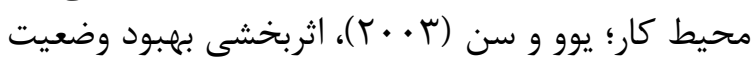

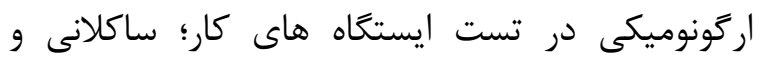

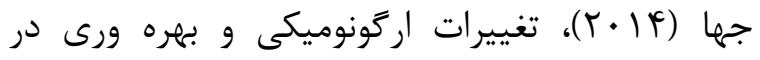

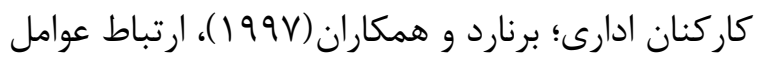

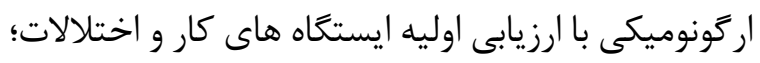

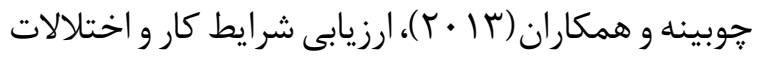

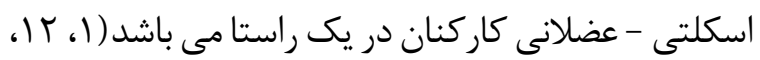

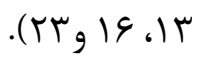
علاوه بر اين، نتايج اين تحقيق نشان إنان مى ده دهد كه

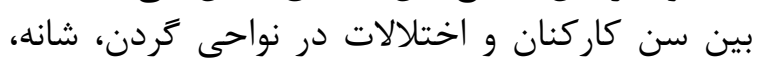

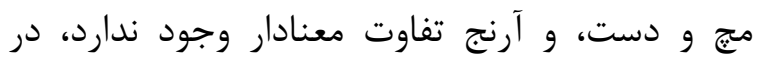

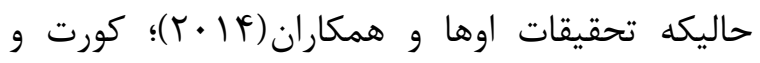

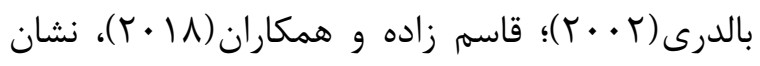

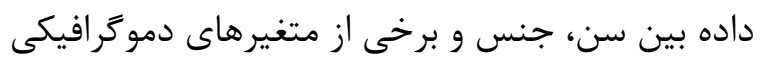




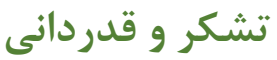

$$
\begin{aligned}
& \text { نويسندكان مراتب تشكر و قدردانى خود را از مساعدات }
\end{aligned}
$$

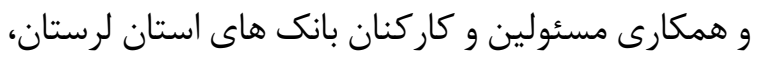

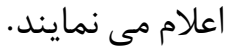

منابع

1. Woods V, Buckle P. Work, inequality and musculoskeletal health. HSE Books Suffolk; 2002.

2. Kaewdok T, Taptagaporn S. G3-3 Ergonomic hazard identification on musculoskeletal discomfort among electronic workers in Thailand. Japanese J Ergon. 2017;53(Supplement2):S462-5.

3. Janwantanakul P, Pensri P, Jiamjarasrangsri V, Sinsongsook T. Prevalence of self-reported musculoskeletal symptoms among office workers. Occup Med (Chic Ill). 2008;58(6):436-8.

4. Abledu JK, Abledu GK. Multiple logistic regression analysis of predictors of musculoskeletal disorder and disability among bank workers in Kumasi. Ghana J Erg. 2012;2:111-5.

5. Oha K, Animägi L, Pääsuke $M$, Coggon $\mathrm{D}$, Merisalu E. Individual and work-related risk factors for musculoskeletal pain: a cross-sectional study among Estonian computer users. BMC Musculoskelet Disord. 2014;15(1):1.

6. Kortt M, Baldry J. The association between musculoskeletal disorders and obesity. Aust Heal Rev. 2002;25(6):207-14.

7. Maiya AG, Bhat VH, Sathiakumar N. Prevalence of Work-Related Wrist and Hand Musculoskeletal Disorders (WMSD) among Computer Users, Karnataka State, India. J Clin Diagnostic Res. 2011;5(3):605-7.

8. Bernaards CM, Bosmans JE, Hildebrandt $\mathrm{VH}$, van Tulder MW, Heymans MW. The cost-effectiveness of a lifestyle physical activity intervention in addition to a work style intervention on recovery from neck and upper limb symptoms and pain reduction in computer workers. Occup Environ Med. 2011;68(4):265-72.

9. Kaliniene G, Ustinaviciene R, Skemiene L, Januskevicius V. Associations between neck musculoskeletal complaints and work related factors among public service computer workers in Kaunas. Int J Occup Med Environ Health. 2013;26(5):670-81.

10. Tantawy SA, Abdul Rahman A, Abdul Ameer M. The relationship between the development of musculoskeletal disorders, body mass index, and academic stress in Bahraini University students. Korean J Pain. 2017;30(2):126-33.

11. Sulaiman SK, Kamalanathan P, Ibrahim AA, Nuhu JM. Musculoskeletal disorders and associated disabilities
كاركنان ادارى؛ طباطبايى و همكاران(Y) • (Y)، ارتباط

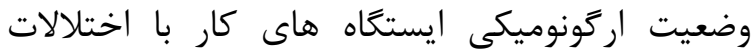

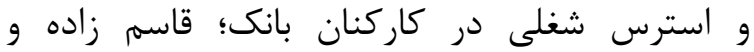

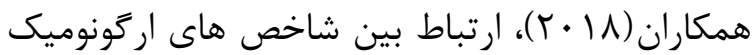

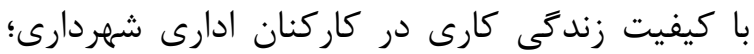

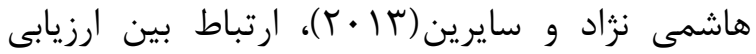

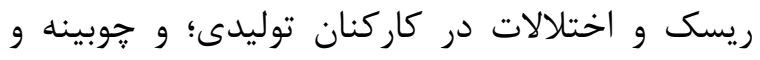

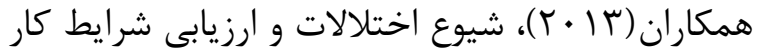

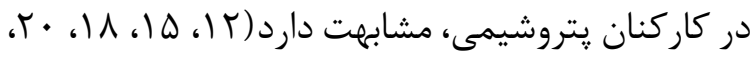

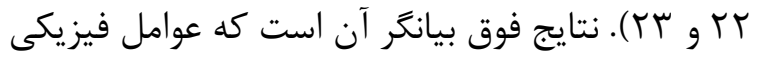

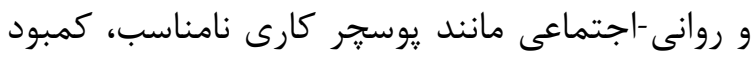

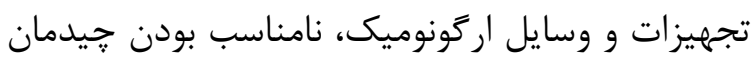

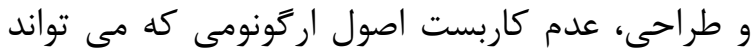

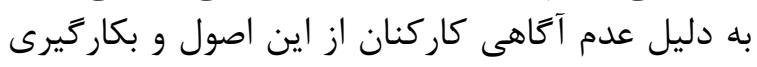

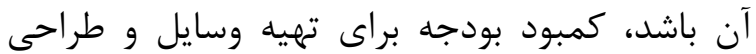

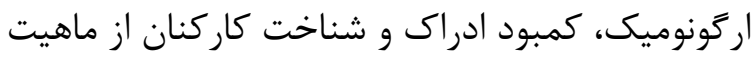

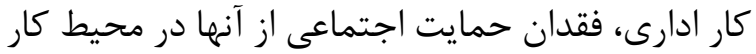

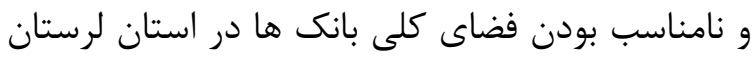

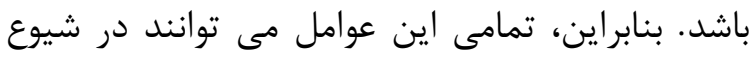

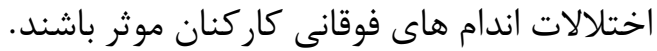

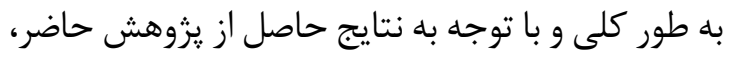

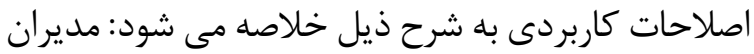

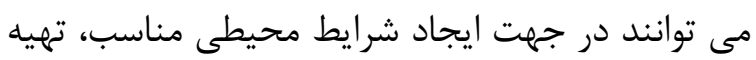

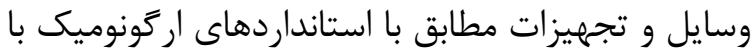

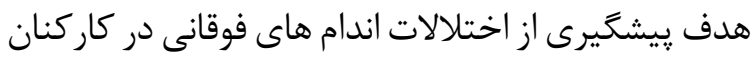

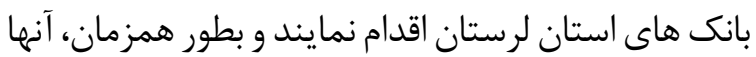

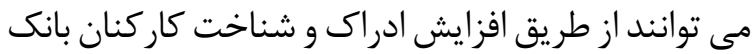

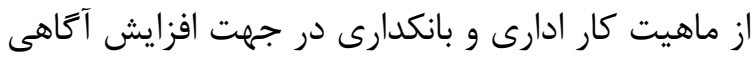

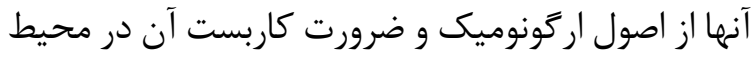

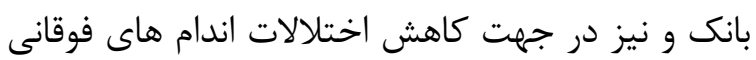

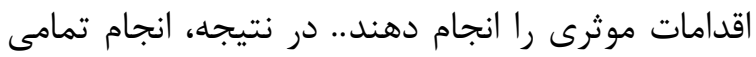

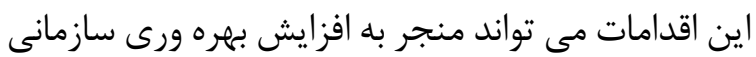
كردد. محدوديت هاى اين تحقيق عبارتند از: اجراى بروهش

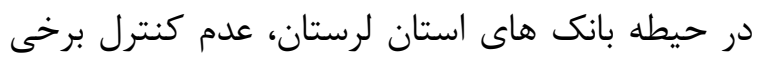
از متغيرهاى مزاحم كه در خارج از محيط كار كار كاركنان

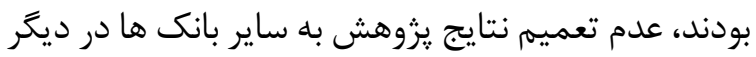
استان هاى كشور. در انتها، يزوهشخَران، اصلاح ايستخاه

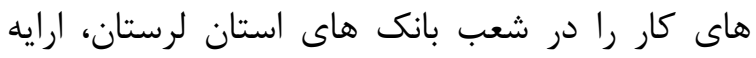

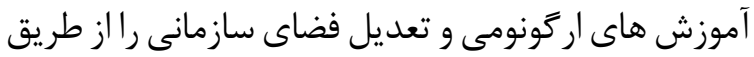

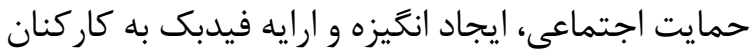
را توصيه مى كنند. 
بر بـ اركونوميك ايستًاه هاى كار ...

The Relationship between the Ergonomic Situation of the Workstations and Musculoskeletal Disorders with the Quality of Work Life and Demographic Variables in the Administrative Staff of the Tehran Municipality. J Ergon. 2018; 5 (3):1-11. [Persian]

21. Choobineh, A. R., Rahimifard, $\mathrm{H}$ and Mahmood khani,S. Musculoskeletal injuries and it's risk factors in administrative workplace. Journal of Iran Occupational Health.2011:8(4),70-81. [Persian]

22. Hashemi Nejad, N., Choobineh, A.R., Rahimifard, H., Haidari, H.R., Tabatabaei, S.H.R. Musculoskeletal disorders risk assessment in small furniture manufacturing workshops. International Journal of Occupational Safety and Ergonomics. 2013.19(2), 275-284.

23. Choobineh, A.R., Daneshmandi, H., Asadi, S., Ahmadi, S. Prevalence of musculoskeletal symptoms and assessment of working conditions in an Iranian Petrochemical Industry. Journal of Health Sciences and Surveillance System. 2013;1(1), 33-4.

24. Choobineh A, Lahmi M, Shahnavaz H, Khani Jazani R, Hosseini M. Musculoskeletal symptoms as related to ergonomic factors in Iranian hand-woven carpet industry and general guidelines for workstation design. Int J Occup Saf Ergon. 2004;10(2):157-68.

25. Lee RD, Nieman DC. Nutritional assessment. 2003. Mc Graw Hill Co New York. 221:227.

26. Cohen AL. Elements of ergonomics programs: a primer based on workplace evaluations of musculoskeletal disorders. DIANE Publishing; 1997.

27. Kuorinka I, Jonsson B, Kilbom A, Vinterberg H, BieringSørensen F, Andersson G, et al. Standardised Nordic questionnaires for the analysis of musculoskeletal symptoms. Appl Ergon. 1987;18(3):233-7.

28. Hersey P, Goldsmith M. A situational approach to performance planning. Train Dev J. 1980;34(11):38.

29. Hatami H, Mirjafari A, Mojadedi JS. An Investigation into the Relationship of Working Life Quality to Organizational Commitment and Productivity in Jahrom Medical Science University. 2011.[Persian]

30. Mohammad A, Ahmad MSA, Reza AA. The relationship between quality of work life and faculty members' productivity in Islamic Azad Universities in district 4 of Iran. Eur J Exp Biol. 2013;3(1):443-8. among bank workers. Int J Res Med Sci. 2017;3(5):11538.

12. Yeow PHP, Sen RN. Quality, productivity, occupational health and safety and cost effectiveness of ergonomic improvements in the test workstations of an electronic factory. Int J Ind Ergon. 2003;32(3):147-63.

13. Saklani A, Jha S. Impact of ergonomic changes on office employee productivity. Saklani, Alok Jha, Shweta(2011) Impact Ergon Chang Off Empl Product Int J Manag Res. 2014;2(1).

14. Helland M, Horgen G, Kvikstad TM, Garthus T, Aarås A. Will musculoskeletal and visual stress change when Visual Display Unit (VDU) operators move from small offices to an ergonomically optimized office landscape? Appl Ergon. 2011;42(6):839-45.

15. Mahmud N, Bahari SF, Zainudin NF. Psychosocial and ergonomics risk factors related to neck, shoulder and back complaints among Malaysia office workers. Int J Soc Sci Humanit. 2014;4(4):260.

16. Bernard BP, Cohen AL, Fine LJ, Gjessing CC, McGlothlin JD. Elements of ergonomics programs; a primer based on workplace evaluations of musculoskeletal disorders. 1997.

17. Falaki S.H, Akbari H, Hannani M, Derakhshan M, Motalebi Kashani M. Prevalence and postural risk factors associated with musculoskeletal disorders among medical laboratory personnel in Kashan in 2012. Iran Occupational Health, Vol. 12, No. 6, Feb-Mar 2016. [Persian]

18. Tabatabaei Yahyaabadi S, Khani Jazani R, Kavousi A, and Bahraini M. Relationship between Ergonomic Workstations with Musculoskeletal Disorders and Job Stress among Staff of Bank in Tehran City. In V. Duffy \& N. Lightner (Eds.). Advances in Human Factors and Ergonomics in Healthcare and Medical Devices, Advances in Intelligent Systems and Computing. Volume 590. USA: Springer; 2017. p.378-387.

19. Tabatabaei Sh, Khani Jazani R, Kavousi A, Azhdardor M. Relationship between Musculoskeletal Disorders and Quality of Life in Employees of Selected Hospitals in Golestan Province. Journal of Health Research in Community. Spring 2017;3(1): 45-56. [Persian]

20. Ghasemzade P, Tabatabaei S, Kavousi A, Sareme M. 